Turkish Journal of Mathematics

http://journals.tubitak.gov.tr/math/
Turk J Math

(2018) 42: $2125-2147$

() TÜBİTAK

doi:10.3906/mat-1705-65

\title{
Parametric nondifferentiable multiobjective fractional programming under
}

$$
(b, \Psi, \Phi, \rho) \text {-univexity }
$$

Tadeusz ANTCZAK*(D), Ram VERMA

Faculty of Mathematics and Computer Science, University of Łódź, Łódź, Poland

Department of Mathematics, University of North Texas, Denton, TX, USA

\begin{tabular}{lllll} 
Received: 16.05 .2017 & $\bullet$ & Accepted/Published Online: 09.04.2018 & Final Version: 27.09 .2018 \\
\hline
\end{tabular}

\begin{abstract}
In this paper, we are concerned with optimality conditions and duality results for nondifferentiable multiobjective fractional programming problems. Parametric necessary optimality conditions are established for such vector optimization problems in which each component of the involved functions is locally Lipschitz. Further, under the introduced concept of nondifferentiable $(b, \Psi, \Phi, \rho)$-univexity, the parametric sufficient optimality conditions are established for a new class of nonconvex multiobjective fractional programming problems. Furthermore, for the considered multiobjective fractional programming problem, its parametric vector dual problem in the sense of Schaible is defined. Then several duality theorems are also established under $(b, \Psi, \Phi, \rho)$-univexity hypotheses.
\end{abstract}

Key words: Parametric multiobjective fractional programming, nondifferentiable vector-valued $(b, \Psi, \Phi, \rho)$-univex function, parametric optimality conditions, parametric duality

\section{Introduction}

In recent years, multiobjective fractional programming problems have received much attention by many authors due to the fact that in many operations research problems the objective functions are quotients of two functions. Therefore, many authors established necessary optimality conditions and employed the conditions to search for optimal solutions as well as duality theorems for such vector optimization problems in the recent past (see, for example, $[1,2,5,6,8-10,12,16,18,20-22,25-31,33,35,37-40,42-44])$. This is a consequence of the fact that multiobjective fractional programming problems have been applied in various fields in different types of objective functions with different constraints including portfolio selection, stock cutting, game theory, and numerous decision problems in management science.

Recently, optimality conditions and duality for nonsmooth multiobjective fractional programs have been studied under various kinds of generalized convexity notions. In [27], Liu established the Kuhn-Tucker necessary and sufficient optimality conditions for an efficient optimum of multiobjective fractional programming problems containing $(F, \rho)$-convex functions and duality results for the defined Bector-type dual problem. Kuk et al. [21] established generalized Karush-Kuhn-Tucker necessary and sufficient optimality conditions and derived duality theorems for nonsmooth multiobjective fractional programming problems containing $V$ - $\rho$-invex functions. In [37], Soleimani-damaneh, using the properties of limiting subdifferential vectors and a separation theorem in convex analysis, derived some necessary and sufficient optimality conditions for the considered nonsmooth frac-

*Correspondence: antczak@math.uni.lodz.pl

2010 AMS Mathematics Subject Classification: 90C32, 90C47, 90C46, 90C26 
tional multiple objective problems. In [20], under the introduced concept of a nearly invex function for locally Lipschitz vector-valued functions, Kim et al. derived generalized sufficient optimality conditions and proved weak and strong duality theorems for the multiobjective fractional optimization problem involving nearly invex functions. Kim [18] introduced the concept of generalized invexity for a fractional function and then he proved the sufficient optimality conditions and several duality results for the considered nonsmooth multiobjective fractional programming problems involving locally Lipschitz functions. Under the introduced generalized $(F, \theta, \rho, d)$-convexity notion, Liu and Feng [28] derived optimality conditions and duality results for a class of nonsmooth multiobjective fractional programming problems. In [33], Nobakhtian considered nonsmooth multiobjective fractional programming problems with mixed constraints and established the optimality conditions and several mixed duality results under various generalized invexity assumptions. Mishra and Upadhyay [30] established necessary and sufficient conditions for a feasible solution to be efficient in a nonsmooth multiobjective fractional programming problem involving $\eta$-pseudolinear functions. They also showed the equivalence between efficiency and proper efficiency under certain boundedness condition and proved various duality results for the corresponding Mond-Weir subgradient type dual problem also under $\eta$-pseudolinearity hypotheses. Lai and Ho [22] studied a subdifferentiable multiobjective fractional programming and established sufficient optimality conditions and parametric duality results under exponential $V$ - $r$-invexity hypotheses. For a bibliography of fractional programming, see Stancu-Minasian [38].

In the paper, we shall establish parametric necessary optimality conditions for the considered nonsmooth multiobjective fractional programming problem with both inequality and equality constraints in which each component of the involved functions is a locally Lipschitz function. Further, we introduce a new concept of generalized convexity, that is, the definition of nondifferentiable $(b, \Psi, \Phi, \rho)$-univexity, which contains many concepts of generalized convexity previously defined in the literature. Then we prove parametric sufficient optimality conditions for a new class of multiobjective fractional programming problems in which the involved functions are $(b, \Psi, \Phi, \rho)$-univex (not necessarily with respect to the same $b, \Psi$ and $b$ ). Furthermore, for the considered nonsmooth multiobjective fractional programming problem, we also define its parametric vector dual problem in the sense of Schaible. Then, also under nondifferentiable $(b, \Psi, \Phi, \rho)$-univexity hypotheses, we prove several duality results between the primal nonsmooth multiobjective fractional programming problem and its parametric Schaible dual problem. In particular, it does not seem that the optimality and duality results have been established previously in the literature for such a large class of nonconvex multiobjective fractional problems with locally Lipschitz functions.

\section{Preliminaries}

In this section, we provide some definitions and some results that we shall use in the sequel.

The following convention for equalities and inequalities will be used in the paper.

For any vectors $x=\left(x_{1}, x_{2}, \ldots, x_{n}\right)^{T}, y=\left(y_{1}, y_{2}, \ldots, y_{n}\right)^{T}$ in $R^{n}$, we define:

(i) $x=y$ if and only if $x_{i}=y_{i}$ for all $i=1,2, \ldots, n$;

(ii) $x>y$ if and only if $x_{i}>y_{i}$ for all $i=1,2, \ldots, n$;

(iii) $x \geqq y \quad$ if and only if $x_{i} \geqq y_{i}$ for all $i=1,2, \ldots, n$;

(iv) $x \geq y$ if and only if $x \geqq y$ and $x \neq y$. 
Throughout this section, $X$ is a nonempty subset of $R^{n}$ and $u$ is an arbitrary given point of $X$.

A function $f: R^{n} \rightarrow R$ is locally Lipschitz at a point $u \in R^{n}$ if there exist scalars $K>0$ and $\varepsilon>0$ such that the following inequality $|f(y)-f(z)| \leqq K\|y-z\|$ holds for all $y, z \in u+\varepsilon B$, where $B$ signifies the open unit ball in $R^{n}$, so that $u+\varepsilon B$ is the open ball of radius $\varepsilon$ about $u$.

Definition 1 [13] The Clarke generalized directional derivative of a locally Lipschitz function $f: X \rightarrow R$ at $u \in X$ in the direction $v \in R^{n}$, denoted $f^{0}(u ; v)$, is given by

$$
f^{0}(u ; v)=\limsup _{\substack{y \rightarrow u \\ \theta \downarrow 0}} \frac{f(y+\theta v)-f(y)}{\theta} .
$$

Definition 2 [13] The Clarke generalized gradient of a locally Lipschitz function $f: X \rightarrow R$ at $u \in X$, denoted $\partial f(u)$, is defined as follows:

$$
\partial f(u)=\left\{\xi \in R^{n}: f^{0}(u ; v) \geqq\langle\xi, v\rangle \text { for all } v \in R^{n}\right\}
$$

Lemma 3 [13] Let $f: X \rightarrow R$ be a locally Lipschitz function on $X$ and let $u$ be an arbitrary point of $X$ and $\lambda \in R$. Then

$$
\partial(\lambda f)(u)=\lambda \partial f(u)
$$

Proposition 4 [13] Let $f_{i}: X \rightarrow R, i=1, \ldots, k$, be locally Lipschitz functions on $X$ and let $u$ be an arbitrary point of $X$. Then

$$
\partial\left(\sum_{i=1}^{k} f_{i}\right)(u) \subseteq \sum_{i=1}^{k} \partial f_{i}(u) .
$$

Equality holds in the above relation if all but at most one of the functions $f_{i}$ are strictly differentiable at $u$.

Corollary 5 [13] For any scalars $\lambda_{i}$, one has

$$
\partial\left(\sum_{i=1}^{k} \lambda_{i} f_{i}\right)(u) \subseteq \sum_{i=1}^{k} \lambda_{i} \partial f_{i}(u)
$$

and equality holds if all but at most one of the $f_{i}$ are strictly differentiable at $u$.

Definition $6\left[\right.$ [7] A function $f: R^{n} \rightarrow R$ is said to be superlinear on $R$ if

i) $f(x+y) \geqq f(x)+f(y), \quad \forall x, y \in R^{n}$,

ii) $f(\lambda x)=\lambda f(x), \forall x \in R^{n}, \forall \lambda \in R, \lambda \geqq 0$.

We now introduce a new concept of generalized convexity, that is, we give the definition of a nondifferentiable vector-valued $(b, \Psi, \Phi, \rho)$-univex function. The introduced concept of a nondifferentiable $(b, \Psi, \Phi, \rho)$ univexity in the vectorial case generalizes the definition of an univex function given by Bector et al. [7] for a differentiable scalar optimization problem and the definition of a nondifferentiable $(\Phi, \rho)$-invex vector-valued function introduced by Antczak [3]. 
ANTCZAK and VERMA/Turk J Math

Definition 7 Let $f: R^{n} \rightarrow R^{p}$ be a vector-valued function such that each its component $f_{i}, i=1, . ., p$, be a locally Lipschitz function, and $\bar{x} \in R^{n}$ be given. If there exist functions $\Psi=\left(\Psi_{1}, \ldots, \Psi_{p}\right): R \rightarrow R^{p}$, $b=\left(b_{1}, \ldots, b_{p}\right): R^{n} \times R^{n} \rightarrow R_{+}^{p}, \Phi: R^{n} \times R^{n} \times R^{n} \times R \rightarrow R$, and $\rho=\left(\rho_{1}, \ldots, \rho_{p}\right) \in R^{p}$ such that, for all $x \in R^{n}, \Phi(x, \bar{x} ;(\cdot, \cdot))$ is convex, $\Phi(x, \bar{x} ;(0, a)) \geqq 0$ for all $a \in R_{+}$, such that the inequalities

$$
b_{i}(x, \bar{x}) \Psi_{i}\left(f_{i}(x)-f_{i}(\bar{x})\right) \geqq \Phi\left(x, \bar{x} ;\left(\xi_{i}, \rho_{i}\right)\right) \quad(>), i=1, \ldots, p
$$

hold for any $\xi_{i} \in \partial f_{i}(\bar{x})$ and all $x \in R^{n},(x \neq \bar{x})$, then $f$ is said to be (strictly) $(b, \Psi, \Phi, \rho)-$ univex at $\bar{x}$ on $R^{n}$. If inequalities (1) is satisfied at each $\bar{x} \in R^{n}$, then $f$ is said to be (strictly) $(b, \Psi, \Phi, \rho)-$ univex on $R^{n}$. If inequalities (1) is satisfied for all $\bar{x} \in X$, where $X$ is a nonempty set of $R^{n}$, then $f$ is said to be (strictly) $(b, \Psi, \Phi, \rho)$-univex at $\bar{x} \in X$ on $X$. Each function $f_{i}, i=1, . ., p$, satisfying (1) is said to be locally Lipschitz (strictly) $\left(b_{i}, \Psi_{i}, \Phi, \rho_{i}\right)$-univex at $\bar{x}$ on $R^{n}$.

Remark 8 Note that the definition of a nondifferentiable $(b, \Psi, \Phi, \rho)$-univex vector-valued function generalizes and extends many other generalized convexity notions previously defined in the literature. Indeed, from Definition $\%$, there are the following special cases:

i) If $\Phi\left(x, \bar{x},\left(\xi_{i}, \rho_{i}\right)\right)=\xi_{i}^{T}(x-\bar{x}), \Psi_{i}(a) \equiv a$ and $b(x, \bar{x}) \equiv 1$ for all $x, \bar{x} \in R^{n}$, then we obtain the definition of a (nondifferentiable) convex function.

ii) If $\Phi\left(x, \bar{x},\left(\xi_{i}, \rho_{i}\right)\right)=\xi_{i}^{T}(x-\bar{x})$ and $\Psi_{i}(a) \equiv a$, then we obtain the definition of a (nondifferentiable) b-convex function.

iii) If $\Phi\left(x, \bar{x},\left(\xi_{i}, \rho_{i}\right)\right)=\xi_{i}^{T} \eta(x, \bar{x})$ for a certain mapping $\eta: R^{n} \times R^{n} \rightarrow R^{n}, \Psi_{i}(a) \equiv a$ and $b(x, \bar{x}) \equiv 1$ for all $x, \bar{x} \in R^{n}$, then we obtain the definition of a (locally Lipschitz) invex function (with respect to the function $\eta$ ) (see Reiland [36] in a nonsmooth scalar case and Kim and Schaible [19] and Lee [23] in the vectorial case).

iv) If $\Phi\left(x, \bar{x},\left(\xi_{i}, \rho_{i}\right)\right)=\xi_{i}^{T} \eta(x, \bar{x})$ for a certain mapping $\eta: R^{n} \times R^{n} \rightarrow R^{n}$, then the definition of $(b, \Psi, \Phi, \rho)$ univexity reduces to the definition of a (locally Lipschitz) univex function (with respect to the function $\eta$ ) (see Bector et al. [7] in a differentiable scalar case).

v) If $\Phi\left(x, \bar{x},\left(\xi_{i}, \rho_{i}\right)\right)=\xi_{i}^{T} \eta(x, \bar{x}), \Psi_{i}(a) \equiv a$, and $\eta: R^{n} \times R^{n} \rightarrow R^{n}$, then we obtain the definition of a nondifferentiable b-invex function (with respect to the function $\eta$ ) (see Li et al. [24]).

vi) If $\Phi\left(x, \bar{x},\left(\xi_{i}, \rho_{i}\right)\right)=\xi_{i}^{T}(x-\bar{x})+\rho_{i}\|x-\bar{x}\|^{2}, \Psi_{i}(a) \equiv a$ and $b(x, \bar{x}) \equiv 1$ for all $x, \bar{x} \in R^{n}$, then $(b, \Psi, \Phi, \rho)$-univexity reduces to the definition of a nonsmooth $\rho$-convex function defined by Vial [41] in the scalar case (see also Zalmai [43] in a nondifferentiable case).

vii) If $\Phi\left(x, \bar{x},\left(\xi_{i}, \rho_{i}\right)\right)=\xi_{i}^{T} \eta(x, \bar{x})+\rho_{i}\|\theta(x, \bar{x})\|^{2}, \Psi_{i}(a) \equiv$ a and $b(x, \bar{x}) \equiv 1$ for all $x, \bar{x} \in R^{n}$, $\eta$ : $R^{n} \times R^{n} \rightarrow R^{n}, \theta: R^{n} \times R^{n} \rightarrow R^{n}, \theta(x, \bar{x}) \neq 0$, whenever $x \neq \bar{x}$, then $\left(b, \Psi_{i}, \Phi, \rho\right)$-univexity reduces to the definition of a nonsmooth $\rho$-invex function (with respect to $\eta$ and $\theta$ ), in the scalar case introduced by Jeyakumar [17] (see also Craven [14], Ahmad [1], and Suneja and Lalitha [40] in the vectorial case). 
viii) If $\Phi\left(x, \bar{x},\left(\xi_{i}, \rho_{i}\right)\right)=\xi_{i}^{T} \eta(x, \bar{x})+\rho_{i}\|x-\bar{x}\|^{2}$ for all $x, \bar{x} \in R^{n}, \eta: R^{n} \times R^{n} \rightarrow R^{n}$, then the definition of $a(b, \Psi, \Phi, \rho)$-univex function reduces to the definition of a nonsmooth $\rho$-univex function (with respect to $\eta$ and $\theta$ ) (see, for example, Mishra [29]).

ix) If $\Phi\left(x, \bar{x},\left(\xi_{i}, \rho_{i}\right)\right)=F\left(x, \bar{x}, \xi_{i}\right)$, where $F(x, \bar{x}, \cdot)$ is a sublinear functional on $R^{n}, \Psi_{i}(a) \equiv a$ and $b(x, \bar{x}) \equiv 1$ for all $x, \bar{x} \in R^{n}$, then the definition of $(b, \Psi, \Phi, \rho)$-univexity reduces to the definition of $F$-convexity introduced by Hanson and Mond [15] in the scalar case.

x) If $\Phi\left(x, \bar{x},\left(\xi_{i}, \rho_{i}\right)\right)=F\left(x, \bar{x}, \xi_{i}\right)+\rho_{i} d^{2}(x, \bar{x})$, where $F(x, \bar{x}, \cdot)$ is a sublinear functional on $R^{n}$, $\Psi_{i}(a) \equiv a$ and $b(x, \bar{x}) \equiv 1$ for all $x, \bar{x} \in R^{n}$, then the definition of $(b, \Psi, \Phi, \rho)$-univexity reduces to the definition of $(F, \rho)$-convexity considered by Mukherjee and Rao [32] in a scalar case, and Liu [27] and Craven [14] in the vectorial case.

xi) If $\Phi\left(x, \bar{x},\left(\xi_{i}, \rho_{i}\right)\right)=\alpha(x, \bar{x}) \xi_{i}^{T} \eta(x, \bar{x})$, where $\eta: R^{n} \times R^{n} \rightarrow R^{n}, \alpha: R^{n} \times R^{n} \rightarrow R_{+} \backslash\{0\}, \alpha(x, \bar{x})=$ $\frac{1}{b(x, \bar{x})}, \Psi_{i}(a) \equiv a$, then $(b, \Psi, \Phi, \rho)$-univexity reduces to the definition of a nonsmooth $\alpha$-invex function (with respect to $\eta$ ) introduced by Mishra et al. [31].

xii) If $\Phi\left(x, \bar{x},\left(\xi_{i}, \rho_{i}\right)\right)=\alpha(x, \bar{x}) \xi_{i}^{T} \eta(x, \bar{x})$, where $\eta: R^{n} \times R^{n} \rightarrow R^{n}, \alpha: R^{n} \times R^{n} \rightarrow R_{+} \backslash\{0\}, \alpha(x, \bar{x})=$ $\frac{1}{b(x, \bar{x})}$, then $(b, \Psi, \Phi, \rho)$-univexity reduces to the definition of a nonsmooth $\alpha$-univex function (with respect to $\eta$ and $\theta$ ) introduced by Jayswal et al. [16].

xiii) If $\Phi\left(x, \bar{x},\left(\xi_{i}, \rho_{i}\right)\right)=F\left(x, \bar{x}, \xi_{i}\right)+\rho_{i} d^{2}(x, \bar{x})$, where $F(x, \bar{x}, \cdot)$ is a sublinear functional on $R^{n}$, $\Psi_{i}(a) \equiv a$, and $d: X \times X \rightarrow R$, then $(b, \Psi, \Phi, \rho)$-univexity notion reduces to $(b, F, \rho)$-convexity, in the smooth vectorial case introduced by Pandian [34].

xiv) If $\Psi_{i}(a) \equiv a$ and $b(x, \bar{x}) \equiv 1$ for all $x, \bar{x} \in R^{n}$, then we obtain the definition of a locally Lipschitz $(\Phi, \rho)$-invex function (see Antczak and Stasiak [4] in a scalar case, and Antczak [3] in a nondifferentiable vectorial case).

In the paper, consider the following multiobjective fractional programming problem:

$$
\begin{gathered}
V \text {-minimize } \varphi(x):=\left(\frac{f_{1}(x)}{q_{1}(x)}, \ldots, \frac{f_{k}(x)}{q_{k}(x)}\right) \\
\text { subject to } \quad \begin{aligned}
g_{j}(x) \leqq 0, j \in J=\{1, \ldots m\}, \\
h_{s}(x)=0, s \in S=\{1, \ldots, p\}, \\
x \in X,
\end{aligned}
\end{gathered}
$$

where $f_{i}: X \rightarrow R, q_{i}: X \rightarrow R, i \in I=\{1, \ldots, k\}, g_{j}: X \rightarrow R, j \in J, h_{s}: X \rightarrow R, s \in S$, are locally Lipschitz functions on a nonempty open convex set $X \subset R^{n}$ and, moreover, $f_{i}(x) \geqq 0, q_{i}(x)>0$, $i \in I$, for all $x \in X$. Let $D:=\left\{x \in X: g_{j}(x) \leqq 0, j \in J, h_{s}(x)=0, s \in S, f_{i}(x) \geqq 0, q_{i}(x)>0, i \in I\right\}$ (assumed to be nonempty) denote the set of all feasible solutions in the considered nonsmooth multiobjective fractional programming problem $(\mathrm{MFP})$. We also denote by $J(x)$ the set of active constraints at $x \in D$, that is, $J(x)=\left\{j \in J: g_{j}(x)=0\right\}$. 
For such multicriterion optimization problems as the considered multiobjective fractional programming problem (MFP), the optimal solution is defined in terms of a (weak) Pareto solution ((weakly) efficient solution) in the following sense:

Definition 9 A feasible point $\bar{x}$ is said to be a weak Pareto solution (weakly efficient solution, weak minimum) for (MFP) if and only if there exists no $x \in D$ such that $\varphi(x)<\varphi(\bar{x})$.

Definition 10 A feasible point $\bar{x}$ is said to be a Pareto solution (efficient solution) for (MFP) if and only if there exists no $x \in D$ such that $\varphi(x) \leq \varphi(\bar{x})$.

\section{Optimality}

In this section, for the considered nonsmooth multiobjective fractional programming problem (MFP), we prove various sufficient optimality conditions under a variety of $(b, \Psi, \Phi, \rho)$-univexity hypotheses.

In order to prove the parametric necessary optimality conditions for the considered multiobjective fractional programming problem (MFP), we use the parametric approach which is based on the method introduced by Crouzeix et al. [11] for minimax fractional programming problems (see also [6]). Then, for the consid-

ered multiobjective fractional programming problem (MFP), we define the associated parametric nonfractional multiobjective programming problem in the parameter $v$ as follows:

$$
\begin{gathered}
V \text {-minimize }\left(f_{1}(x)-v_{1} q_{1}(x), \ldots, f_{k}(x)-v_{k} q_{k}(x)\right) \\
\text { subject to } \quad g_{j}(x) \leqq 0, j \in J=\{1, \ldots m\}, \\
h_{s}(x)=0, s \in S=\{1, \ldots, p\} \\
x \in X .
\end{gathered}
$$

For the auxiliary multiobjective programming problem (MP) defined above, the following result is true:

Lemma 11 Let $\bar{x} \in D$ be a weak Pareto solution of the considered multiobjective fractional programming problem (MFP). Then $\bar{x}$ is also a weak Pareto solution in the nonfractional multiobjective programming problem (MP) with $\bar{v}_{i}=\varphi_{i}(\bar{x})$, that $i s, \bar{v}_{i}=\frac{f_{i}(\bar{x})}{q_{i}(\bar{x})}, i \in I$.

Theorem 12 (Parametric necessary optimality conditions). Let $\bar{x} \in D$ be a weak Pareto solution of the considered multiobjective fractional programming problem (MFP) with $\bar{v}=\varphi(\bar{x})$ and the suitable constraint qualification (for example, the generalized Slater constraint qualification) be satisfied at $\bar{x}$. Then there exist $\bar{\lambda} \in R^{k}, \bar{\mu} \in R^{m}$, and $\bar{\omega} \in R^{p}$ such that

$$
\begin{gathered}
0 \in \sum_{i=1}^{k} \bar{\lambda}_{i} \partial\left(f_{i}(\bar{x})-\bar{v}_{i} q_{i}(\bar{x})\right)+\sum_{j=1}^{m} \bar{\mu}_{j} \partial g_{j}(\bar{x})+\sum_{s=1}^{p} \bar{\omega}_{s} \partial h_{s}(\bar{x}), \\
\bar{\mu}_{j} g_{j}(\bar{x})=0, j \in J, \\
\bar{\lambda}_{i} \geq 0, i \in I, \sum_{i=1}^{k} \bar{\lambda}_{i}=1, \bar{\mu}_{j} \geqq 0, j \in J .
\end{gathered}
$$


Proof Let $\bar{x} \in D$ be a weak Pareto solution of the considered multiobjective fractional programming problem (MFP). Hence, by Lemma 11, $\bar{x}$ is also a weak Pareto solution of the associated parametric nonfractional multiobjective programming problem (MP). Further, by Theorem 2.1 [3], there exist Lagrange multipliers $\bar{\lambda} \in R^{k}, \bar{\mu} \in R^{m}$ and $\bar{\omega} \in R^{p}$ such that the above conditions (2)-(4) are fulfilled. This completes the proof of this theorem.

The following result follows directly from Theorem 12 .

Corollary 13 (Parametric necessary optimality conditions). Let $\bar{x} \in D$ be a weak Pareto solution in the considered multiobjective fractional programming problem (MFP) with $\bar{v}=\varphi(\bar{x})$ and the suitable constraint qualification (for example, the generalized Slater constraint qualification) be satisfied at $\bar{x}$. Then there exist $\bar{\lambda} \in R^{k}, \bar{\mu} \in R^{m}$, and $\bar{\omega} \in R^{p}$ such that

$$
\begin{gathered}
0 \in \sum_{i=1}^{k} \bar{\lambda}_{i}\left(\partial f_{i}(\bar{x})-\bar{v}_{i} \partial q_{i}(\bar{x})\right)+\sum_{j=1}^{m} \bar{\mu}_{j} \partial g_{j}(\bar{x})+\sum_{s=1}^{p} \bar{\omega}_{s} \partial h_{s}(\bar{x}), \\
\bar{\mu}_{j} g_{j}(\bar{x})=0, j \in J \\
\bar{\lambda}_{i} \geq 0, i \in I, \sum_{i=1}^{k} \bar{\lambda}_{i}=1, \bar{\mu}_{j} \geqq 0, j \in J .
\end{gathered}
$$

Proof Let $\bar{x} \in D$ be a weak Pareto solution in the considered multiobjective fractional programming problem (MFP). Hence, there exist $\bar{\lambda} \in R^{k}, \bar{\mu} \in R^{m}$, and $\bar{\omega} \in R^{p}$ such that the parametric necessary optimality conditions (2)-(4) are fulfilled at $\bar{x}$. Therefore, it is sufficient to prove that the condition (5) follows from (2). Indeed, by Lemma 3 and Proposition 4, it follows that $\partial\left(f_{i}(\bar{x})-\bar{v}_{i} q_{i}(\bar{x})\right) \subseteq \partial f_{i}(\bar{x})-\bar{v}_{i} \partial q_{i}(\bar{x}), i \in I$. This completes the proof of this corollary.

Theorem 14 Let $\bar{x} \in D, \bar{v}=\varphi(\bar{x})$ and the parametric necessary optimality be satisfied at $\bar{x}$ with Lagrange multipliers $\bar{\lambda} \in R^{k}, \bar{\mu} \in R^{m}, \bar{\omega} \in R^{p}$. Further, assume that either one of the following two sets of hypotheses is satisfied:

A) a) for each $i=1, \ldots, k, f_{i}(\cdot)-\bar{v}_{i} q_{i}(\cdot)$ is $\left(b_{i}, \Psi_{i}, \Phi, \rho_{i}\right)$-univex at $\bar{x}$ on $D$ and $a<0 \Longrightarrow \Psi_{i}(a)<0$;

b) for each $j \in J(\bar{x}), g_{j}(\cdot)$ is $\left(b_{g_{j}}, \Psi_{g_{j}}, \Phi, \rho_{g_{j}}\right)$-univex at $\bar{x}$ on $D$ and $a \leqq 0 \Longrightarrow \Psi_{g_{j}}(a) \leqq 0$;

c) for each $s \in S^{+}(\bar{x}):=\{s \in S: \bar{\omega}>0\}, h_{s}(\cdot)$ is $\left(b_{h_{s}}, \Psi_{h_{s}}, \Phi, \rho_{h_{s}}^{+}\right)$-univex at $\bar{x}$ on $D$ and $\left(\Psi_{h_{s}}(0)=0\right.$ or $\left.a \leqq 0 \Longrightarrow \Psi_{h_{s}}(a) \leqq 0\right)$;

d) for each $s \in S^{-}(\bar{x}):=\{s \in S: \bar{\omega}<0\}, \quad-h_{s}(\cdot)$ is $\left(b_{h_{s}}, \Psi_{h_{s}}, \Phi, \rho_{h_{s}}^{-}\right)$-univex at $\bar{x}$ on $D$ and $\left(\Psi_{h_{s}}(0)=0\right.$ or $\left.a \leqq 0 \Longrightarrow \Psi_{h_{s}}(a) \leqq 0\right)$;

e) $\sum_{i=1}^{k} \bar{\lambda}_{i} \rho_{i}+\sum_{j \in J(\bar{x})} \bar{\mu}_{j} \rho_{g_{j}}+\sum_{s \in S^{+}(\bar{x})} \bar{\omega}_{s} \rho_{h_{s}}^{+}-\sum_{s \in S^{-}(\bar{x})} \bar{\omega}_{s} \rho_{h_{s}}^{-} \geqq 0$.

B) a) for each $i=1, \ldots, k, f_{i}(\cdot)$ is $\left(b_{i}, \Psi_{i}, \Phi, \rho_{f_{i}}\right)$-univex at $\bar{x}$ on $D$ and $-q_{i}(\cdot)$ are $\left(b_{i}, \Psi_{i}, \Phi, \rho_{i}\right)$-univex at $\bar{x}$ on $D, \Psi_{i}$ is a superlinear function and $a<0 \Longrightarrow \Psi_{i}(a)<0$; 
b) for each $j \in J(\bar{x}), g_{j}(\cdot)$ is $\left(b_{g_{j}}, \Psi_{g_{j}}, \Phi, \rho_{g_{j}}\right)$-univex at $\bar{x}$ on $D$ and $a \leqq 0 \Longrightarrow \Psi_{g_{j}}(a) \leqq 0$;

c) for each $s \in S^{+}(\bar{x}), h_{s}(\cdot)$ is $\left(b_{h_{s}}, \Psi_{h_{s}}, \Phi, \rho_{h_{s}}^{+}\right)$-univex at $\bar{x}$ on $D$ and $\left(\Psi_{h_{s}}(0)=0\right.$ or $\left.a \leqq 0 \Longrightarrow \Psi_{h_{s}}(a) \leqq 0\right)$;

d) for each $s \in S^{-}(\bar{x}),-h_{s}(\cdot)$ is $\left(b_{h_{s}}, \Psi_{h_{s}}, \Phi, \rho_{h_{s}}^{-}\right)$-univex at $\bar{x}$ on $D$ and $\left(\Psi_{h_{s}}(0)=0\right.$ or $\left.a \leqq 0 \Longrightarrow \Psi_{h_{s}}(a) \leqq 0\right)$;

e) $\sum_{i=1}^{k} \bar{\lambda}_{i}\left(\rho_{f_{i}}+\bar{v}_{i} \rho_{q_{i}}\right)+\sum_{j \in J(\bar{x})} \bar{\mu}_{j} \rho_{g_{j}}+\sum_{s \in S^{+}(\bar{x})} \bar{\omega}_{s} \rho_{h_{s}}^{+}-\sum_{s \in S^{-}(\bar{x})} \bar{\omega}_{s} \rho_{h_{s}}^{-} \geqq 0$.

Then $\bar{x}$ is a weak Pareto solution of the considered nonsmooth multiobjective fractional programming problem (MFP).

Proof We now establish this theorem under hypothesis A). Suppose, contrary to the result, that $\bar{x}$ is not a weak Pareto optimal solution of the problem (MFP). Then, by Definition 9, there exists $\widetilde{x} \in D$ such that

$$
\varphi(\widetilde{x})<\varphi(\bar{x}) .
$$

Hence, by the definition of $\varphi$ and $\bar{v}=\varphi(\bar{x}),(8)$ implies

$$
f_{i}(\widetilde{x})-\bar{v}_{i} q_{i}(\widetilde{x})<f_{i}(\bar{x})-\bar{v}_{i} q_{i}(\bar{x}), i \in I .
$$

By hypothesis a), each function $f_{i}(\cdot)-\bar{v}_{i} q_{i}(\cdot), i \in I$, is locally Lipschitz $\left(b_{i}, \Psi_{i}, \Phi, \rho_{i}\right)$-univex at $\bar{x}$ on $D$. Thus, by Definition 7, it follows that the inequalities

$$
b_{i}(\widetilde{x}, \bar{x}) \Psi_{i}\left(f_{i}(\widetilde{x})-\bar{v}_{i} q_{i}(\widetilde{x})-\left(f_{i}(\bar{x})-\bar{v}_{i} q_{i}(\bar{x})\right)\right) \geqq \Phi\left(\widetilde{x}, \bar{x},\left(\xi_{i}, \rho_{i}\right)\right), i \in I,
$$

hold for any $\xi_{i} \in \partial\left(f_{i}-\bar{v}_{i} q_{i}\right)(\bar{x}), i \in I$, respectively. Hence, by $b_{i}(\widetilde{x}, \bar{x})>0, i \in I$, and hypothesis a), inequalities (10) imply, respectively,

$$
\Phi\left(\widetilde{x}, \bar{x},\left(\xi_{i}, \rho_{i}\right)\right)<0, \quad i \in I .
$$

Multiplying each inequality (11) by the corresponding Lagrange multiplier $\bar{\lambda}_{i}$ and then adding both sides of the resulting inequalities, we get that the inequality

$$
\sum_{i=1}^{k} \bar{\lambda}_{i} \Phi\left(\widetilde{x}, \bar{x},\left(\xi_{i}, \rho_{i}\right)\right)<0
$$

holds for any $\xi_{i} \in \partial\left(f_{i}-\bar{v}_{i} q_{i}\right)(\bar{x}), i \in I$. By $\widetilde{x} \in D$ and $\bar{x} \in D$, it follows that

$$
g_{j}(\widetilde{x})-g_{j}(\bar{x}) \leqq 0, \quad j \in J(\bar{x})
$$

By hypothesis b), the above inequalities yield, respectively,

$$
b_{g_{j}}(\widetilde{x}, \bar{x}) \Psi_{g_{j}}\left(g_{j}(\widetilde{x})-g_{j}(\bar{x})\right) \leqq 0, \quad j \in J(\bar{x}) .
$$

Since each function $g_{j}, j \in J(\bar{x})$, is locally Lipschitz $\left(b_{g_{j}}, \Psi_{g_{j}}, \Phi, \rho_{g_{j}}\right)$-univex at $\bar{x}$ on $D$, by Definition 7 , it follows that the inequalities

$$
b_{g_{j}}(\widetilde{x}, \bar{x}) \Psi_{g_{j}}\left(g_{j}(\widetilde{x})-g_{j}(\bar{x})\right) \geqq \Phi\left(\widetilde{x}, \bar{x},\left(\zeta_{j}, \rho_{g_{j}}\right)\right), \quad j \in J(\bar{x}),
$$


hold for any $\zeta_{j} \in \partial g_{j}(\bar{x}), j \in J(\bar{x})$, respectively. Combining (13) and (14), we get, respectively,

$$
\Phi\left(\widetilde{x}, \bar{x},\left(\zeta_{j}, \rho_{g_{j}}\right)\right) \leqq 0, \quad j \in J(\bar{x})
$$

Multiplying each inequality (15) by the corresponding Lagrange multiplier $\bar{\mu}_{j} \geqq 0, j \in J(\bar{x})$, and then adding both sides of the resulting inequalities, we get that the inequality

$$
\sum_{j \in J(\bar{x})} \bar{\mu}_{j} \Phi\left(\widetilde{x}, \bar{x},\left(\zeta_{j}, \rho_{g_{j}}\right)\right) \leqq 0
$$

holds for any $\zeta_{j} \in \partial g_{j}(\bar{x}), j \in J(\bar{x})$. Using again the feasibility of $\widetilde{x}$ and $\bar{x}$ in the problem (MFP), we have

$$
h_{s}(\widetilde{x})-h_{s}(\bar{x})=0, s \in S .
$$

By hypotheses c) and d), the above inequalities yield, respectively,

$$
\begin{gathered}
b_{h_{s}}(\widetilde{x}, \bar{x}) \Psi_{h_{s}}\left(h_{s}(\widetilde{x})-h_{s}(\bar{x})\right) \leqq 0, \quad s \in S^{+}(\bar{x}), \\
b_{h_{s}}(\widetilde{x}, \bar{x}) \Psi_{h_{s}}\left(-h_{s}(\widetilde{x})+h_{s}(\bar{x})\right) \leqq 0, \quad s \in S^{-}(\bar{x}) .
\end{gathered}
$$

Since each function $h_{s}, s \in S^{+}(\bar{x})$, is locally Lipschitz $\left(b_{h_{s}}, \Psi_{h_{s}}, \Phi, \rho_{h_{s}}^{+}\right)$-univex at $\bar{x}$ on $D$ and each function $-h_{s}, s \in S^{-}(\bar{x})$, is locally Lipschitz $\left(b_{h_{s}}, \Psi_{h_{s}}, \Phi, \rho_{h_{s}}^{-}\right)$-univex at $\bar{x}$ on $D$, by Definition 7 , it follows that the following inequalities

$$
\begin{gathered}
b_{h_{s}}(\widetilde{x}, \bar{x}) \Psi_{h_{s}}\left(h_{s}(\widetilde{x})-h_{s}(\bar{x})\right) \geqq \Phi\left(\widetilde{x}, \bar{x},\left(\varsigma_{s}, \rho_{h_{s}}^{+}\right)\right), \quad s \in S^{+}(\bar{x}), \\
b_{h_{s}}(\widetilde{x}, \bar{x}) \Psi_{h_{s}}\left(-h_{s}(\widetilde{x})+h_{s}(\bar{x})\right) \geqq \Phi\left(\widetilde{x}, \bar{x},\left(-\varsigma_{s}, \rho_{h_{s}}^{-}\right)\right), \quad s \in S^{-}(\bar{x})
\end{gathered}
$$

hold for any $\varsigma_{s} \in \partial h_{s}(\bar{x}), s \in S^{+}(\bar{x})$ and $-\varsigma_{s} \in \partial\left(-h_{s}\right)(\bar{x}), s \in S^{-}(\bar{x})$, respectively. Combining (17) and $(18),(19)$ and (20) we get, respectively,

$$
\begin{gathered}
\Phi\left(\widetilde{x}, \bar{x},\left(\varsigma_{s}, \rho_{h_{s}}^{+}\right)\right) \leqq 0, \quad s \in S^{+}(\bar{x}), \\
\Phi\left(\widetilde{x}, \bar{x},\left(-\varsigma_{s}, \rho_{h_{s}}^{-}\right)\right) \leqq 0, \quad s \in S^{-}(\bar{x}) .
\end{gathered}
$$

Multiplying each inequality (21) by the corresponding Lagrange multiplier $\bar{\omega}_{s}>0, s \in S^{+}(\bar{x})$, each inequality (22) by $-\bar{\omega}_{s}>0, s \in S^{-}(\bar{x})$, and then adding both sides of the resulting inequalities, we get that the inequalities

$$
\begin{gathered}
\sum_{s \in S^{+}(\bar{x})} \bar{\omega}_{s} \Phi\left(\widetilde{x}, \bar{x},\left(\varsigma_{s}, \rho_{h_{s}}^{+}\right)\right) \leqq 0, \\
\sum_{s \in S^{-}(\bar{x})}\left(-\bar{\omega}_{s}\right) \Phi\left(\widetilde{x}, \bar{x},\left(-\varsigma_{s}, \rho_{h_{s}}^{-}\right)\right) \leqq 0
\end{gathered}
$$


hold for any $\varsigma_{s} \in \partial h_{s}(\bar{x}), s \in S^{+}(\bar{x})$ and $-\varsigma_{s} \in \partial\left(-h_{s}\right)(\bar{x}), s \in S^{-}(\bar{x})$, respectively. Combining (12), (16), (23), and (24), we have

$$
\begin{gathered}
\sum_{i=1}^{k} \bar{\lambda}_{i} \Phi\left(\widetilde{x}, \bar{x},\left(\xi_{i}, \rho_{i}\right)\right)+\sum_{j \in J(\bar{x})} \bar{\mu}_{j} \Phi\left(\widetilde{x}, \bar{x},\left(\zeta_{j}, \rho_{g_{j}}\right)\right)+ \\
\sum_{s \in S^{+}(\bar{x})} \bar{\omega}_{s} \Phi\left(\widetilde{x}, \bar{x},\left(\varsigma_{s}, \rho_{h_{s}}^{+}\right)\right)+\sum_{s \in S^{-}(\bar{x})}\left(-\bar{\omega}_{s}\right) \Phi\left(\widetilde{x}, \bar{x},\left(-\varsigma_{s}, \rho_{h_{s}}^{-}\right)\right)<0 .
\end{gathered}
$$

Let us denote by

$$
\begin{gathered}
A=\sum_{i=1}^{k} \bar{\lambda}_{i}+\sum_{j=1}^{m} \bar{\mu}_{j}+\sum_{s=1}^{p} \bar{\omega}_{s}, \\
\bar{\alpha}_{i}=\frac{\bar{\lambda}_{i}}{A}, i \in I, \\
\bar{\beta}_{j}=\frac{\bar{\mu}_{j}}{A}, j \in J, \\
\bar{\gamma}_{s}^{+}=\frac{\bar{\omega}_{s}}{A}, s \in S^{+}(\bar{x}), \\
\bar{\gamma}_{s}^{-}=\frac{-\bar{\omega}_{s}}{A}, s \in S^{-}(\bar{x}) .
\end{gathered}
$$

Then, by the Karush-Kuhn-Tucker necessary optimality condition (4), it follows that $\bar{\alpha}:=\left(\bar{\alpha}_{1}, \ldots, \bar{\alpha}_{k}\right) \geq 0$, $0 \leqq \bar{\alpha}_{i} \leqq 1, i \in I$, and at least one $\bar{\alpha}_{i}>0, \bar{\beta}_{j}=\left(\bar{\beta}_{1}, \ldots, \bar{\beta}_{m}\right) \geqq 0,0 \leqq \bar{\beta}_{j} \leqq 1, j \in J, 0 \leqq \bar{\gamma}_{s}^{+} \leqq 1, s \in S^{+}(\bar{x})$, $0 \leqq \bar{\gamma}_{s}^{-} \leqq 1, s \in S^{-}(\bar{x}), A>0$, and, moreover, $\sum_{i=1}^{k} \bar{\alpha}_{i}+\sum_{j=1}^{m} \bar{\beta}_{j}+\sum_{s \in S^{+}(\bar{x})} \bar{\gamma}_{s}^{+}+\sum_{s \in S^{-}(\bar{x})} \bar{\gamma}_{s}^{-}=1$. Using (26)-(30) in (25), we get

$$
\begin{gathered}
\sum_{i=1}^{k} \bar{\alpha}_{i} \Phi\left(\widetilde{x}, \bar{x},\left(\xi_{i}, \rho_{f_{i}}\right)\right)+\sum_{j \in J(\bar{x})} \bar{\beta}_{j} \Phi\left(\widetilde{x}, \bar{x},\left(\zeta_{j}, \rho_{g_{j}}\right)\right)+ \\
\sum_{s \in S^{+}(\bar{x})} \bar{\gamma}_{s}^{+} \Phi\left(\widetilde{x}, \bar{x},\left(\varsigma_{s}, \rho_{h_{s}}^{+}\right)\right)+\sum_{s \in S^{-}(\bar{x})} \bar{\gamma}_{s}^{-} \Phi\left(\widetilde{x}, \bar{x},\left(-\varsigma_{s}, \rho_{h_{s}}^{-}\right)\right)<0 .
\end{gathered}
$$

By Definition 7 , it follows that $\Phi(\widetilde{x}, \bar{x}, \cdot)$ is a convex function on $R^{n+1}$. Thus, by the definition of convexity, (31) yields

$$
\begin{gathered}
\Phi\left(\widetilde{x}, \bar{x},\left(\sum_{i=1}^{k} \bar{\alpha}_{i} \xi_{i}+\sum_{j \in J(\bar{x})} \bar{\beta}_{j} \zeta_{j}+\sum_{s \in S^{+}(\bar{x})} \bar{\gamma}_{s}^{+} \varsigma_{s}+\sum_{s \in S^{-}(\bar{x})} \bar{\gamma}_{s}^{-}\left(-\varsigma_{s}\right),\right.\right. \\
\left.\left.\sum_{i=1}^{k} \bar{\alpha}_{i} \rho_{i}+\sum_{j \in J(\bar{x})} \bar{\beta}_{j} \rho_{g_{j}}+\sum_{s \in S^{+}(\bar{x})} \bar{\gamma}_{s}^{+} \rho_{h_{s}}^{+}+\sum_{s \in S^{-}(\bar{x})} \bar{\gamma}_{s}^{-} \rho_{h_{s}}^{-}\right)\right)<0 .
\end{gathered}
$$


Using (26)-(30) in the above inequality, we get

$$
\begin{aligned}
\Phi\left(\widetilde{x}, \bar{x}, \frac{1}{A}\left(\sum_{i=1}^{k} \bar{\lambda}_{i} \xi_{i}+\sum_{j \in J(\bar{x})} \bar{\mu}_{j} \zeta_{j}+\sum_{s \in S^{+}(\bar{x}) \cup S^{-}(\bar{x})} \bar{\omega}_{s} \varsigma_{s}\right.\right. \\
\left.\left.\quad \sum_{i=1}^{k} \bar{\lambda}_{i} \rho_{i}+\sum_{j \in J(\bar{x})} \bar{\mu}_{j} \rho_{g_{j}}+\sum_{s \in S^{+}(\bar{x})} \bar{\omega}_{s} \rho_{h_{s}}^{+}-\sum_{s \in S^{-}(\bar{x})} \bar{\omega}_{s} \rho_{h_{s}}^{-}\right)\right)<0 .
\end{aligned}
$$

Taking into account the Lagrange multipliers $\bar{\mu}_{j}=0, j \notin J(\bar{x}), \bar{\omega}_{s}=0, s \notin S^{+}(\bar{x}) \cup S^{-}(\bar{x}),(32)$ implies that the inequality

$$
\begin{aligned}
\Phi & \left(\widetilde{x}, \bar{x}, \frac{1}{A}\left(\sum_{i=1}^{k} \bar{\lambda}_{i} \xi_{i}+\sum_{j=1}^{m} \bar{\mu}_{j} \zeta_{j}+\sum_{s=1}^{p} \bar{\omega}_{s} \varsigma_{s}\right.\right. \\
& \left.\left.\sum_{i=1}^{k} \bar{\lambda}_{i} \rho_{i}+\sum_{j \in J(\bar{x})} \bar{\mu}_{j} \rho_{g_{j}}+\sum_{s \in S^{+}(\bar{x})} \bar{\omega}_{s} \rho_{h_{s}}^{+}-\sum_{s \in S^{-}(\bar{x})} \bar{\omega}_{s} \rho_{h_{s}}^{-}\right)\right)<0
\end{aligned}
$$

holds for any $\xi_{i} \in \partial\left(f_{i}-\bar{v}_{i} q_{i}\right)(\bar{x}), i \in I, \zeta_{j} \in \partial g_{j}(\bar{x}), j \in J, \varsigma_{s} \in \partial h_{s}(\bar{x}), s \in S$. Hence, by the KarushKuhn-Tucker necessary optimality condition (2), we have

$$
\Phi\left(\widetilde{x}, \bar{x}, \frac{1}{A}\left(0, \sum_{i=1}^{k} \bar{\lambda}_{i} \rho_{i}+\sum_{j \in J(\bar{x})} \bar{\mu}_{j} \rho_{g_{j}}+\sum_{s \in S^{+}(\bar{x})} \bar{\omega}_{s} \rho_{h_{s}}^{+}-\sum_{s \in S^{-}(\bar{x})} \bar{\omega}_{s} \rho_{h_{s}}^{-}\right)\right)<0 .
$$

By hypothesis d), it follows that $\sum_{i=1}^{k} \bar{\lambda}_{i} \rho_{i}+\sum_{j \in J(\bar{x})} \bar{\mu}_{j} \rho_{g_{j}}+\sum_{s \in S^{+}(\bar{x})} \bar{\omega}_{s} \rho_{h_{s}}^{+}-\sum_{s \in S^{-}(\bar{x})} \bar{\omega}_{s} \rho_{h_{s}}^{-} \geqq 0$. Thus, by Definition 7, the following inequality

$$
\Phi\left(\widetilde{x}, \bar{x}, \frac{1}{A}\left(0, \sum_{i=1}^{k} \bar{\lambda}_{i} \rho_{i}+\sum_{j \in J(\bar{x})} \bar{\mu}_{j} \rho_{g_{j}}+\sum_{s \in S^{+}(\bar{x})} \bar{\omega}_{s} \rho_{h_{s}}^{+}-\sum_{s \in S^{-}(\bar{x})} \bar{\omega}_{s} \rho_{h_{s}}^{-}\right)\right) \geqq 0
$$

holds, contradicting (33). This means that $\bar{x}$ is a weak Pareto solution of the problem (MFP) and completes the proof of this theorem under hypothesis A).

The proof of the theorem under hypothesis B) is similar. Namely, the parametric necessary optimality conditions (5)-(7) should be used in place of the parametric necessary optimality conditions (2)-(4), which have been used in the proof of this theorem under hypothesis A).

Under stronger hypotheses imposed on the objective functions, the following result is true:

Theorem 15 Let $\bar{x} \in D, \bar{v}=\varphi(\bar{x})$ and the Karush-Kuhn-Tucker necessary optimality be satisfied at $\bar{x}$ with Lagrange multipliers $\bar{\lambda} \in R^{k}, \bar{\mu} \in R^{m}, \bar{\omega} \in R^{p}$. Further, assume that either one of the following two sets of hypotheses are fulfilled:

A) a) for each $i=1, \ldots, k, f_{i}(\cdot)-\bar{v}_{i} q_{i}(\cdot)$ is strictly $\left(b_{i}, \Psi_{i}, \Phi, \rho_{i}\right)$-univex at $\bar{x}$ on $D$ and $a \leqq 0 \Longrightarrow \Psi_{i}(a) \leqq$ 0 ; 
b) for each $j \in J(\bar{x}), g_{j}(\cdot)$ is $\left(b_{g_{j}}, \Psi_{g_{j}}, \Phi, \rho_{g_{j}}\right)$-univex at $\bar{x}$ on $D$ and $a \leqq 0 \Longrightarrow \Psi_{g_{j}}(a) \leqq 0$;

c) for each $s \in S^{+}(\bar{x}), h_{s}(\cdot)$ is $\left(b_{h_{s}}, \Psi_{h_{s}}, \Phi, \rho_{h_{s}}^{+}\right)$-univex at $\bar{x}$ on $D$ and $\left(\Psi_{h_{s}}(0)=0\right.$ or $a \leqq 0$ $\left.\Longrightarrow \Psi_{h_{s}}(a) \leqq 0\right)$;

d) for each $s \in S^{-}(\bar{x}),-h_{s}(\cdot)$ is $\left(b_{h_{s}}, \Psi_{h_{s}}, \Phi, \rho_{h_{s}}^{-}\right)$-univex at $\bar{x}$ on $D$ and $\left(\Psi_{h_{s}}(0)=0\right.$ or $a \leqq 0$ $\left.\Longrightarrow \Psi_{h_{s}}(a) \leqq 0\right)$;

e) $\sum_{i=1}^{k} \bar{\lambda}_{i} \rho_{i}+\sum_{j \in J(\bar{x})} \bar{\mu}_{j} \rho_{g_{j}}+\sum_{s \in S^{+}(\bar{x})} \bar{\omega}_{s} \rho_{h_{s}}^{+}-\sum_{s \in S^{-}(\bar{x})} \bar{\omega}_{s} \rho_{h_{s}}^{-} \geqq 0$.

B) a) for each $i=1, \ldots, k, f_{i}(\cdot)$ is strictly $\left(b_{i}, \Psi_{i}, \Phi, \rho_{i}\right)$-univex at $\bar{x}$ on $D$ and $-q_{i}(\cdot)$ is strictly $\left(b_{i}, \Psi_{i}, \Phi, \rho_{i}\right)$-univex at $\bar{x}$ on $D, \Psi_{i}$ is a superlinear function and $a \leqq 0 \Longrightarrow \Psi_{i}(a) \leqq 0$;

b) for each $j \in J(\bar{x}), g_{j}(\cdot)$ is $\left(b_{g_{j}}, \Psi_{g_{j}}, \Phi, \rho_{g_{j}}\right)$-univex at $\bar{x}$ on $D$ and $a \leqq 0 \Longrightarrow \Psi_{g_{j}}(a) \leqq 0$;

c) for each $s \in S^{+}(\bar{x}), h_{s}(\cdot)$ is $\left(b_{h_{s}}, \Psi_{h_{s}}, \Phi, \rho_{h_{s}}^{+}\right)$-univex at $\bar{x}$ on $D$ and $\left(\Psi_{h_{s}}(0)=0\right.$ or $a \leqq 0$ $\left.\Longrightarrow \Psi_{h_{s}}(a) \leqq 0\right)$;

d) for each $s \in S^{-}(\bar{x}),-h_{s}(\cdot)$ is $\left(b_{h_{s}}, \Psi_{h_{s}}, \Phi, \rho_{h_{s}}^{-}\right)$-univex at $\bar{x}$ on $D$ and $\left(\Psi_{h_{s}}(0)=0\right.$ or $a \leqq 0$ $\left.\Longrightarrow \Psi_{h_{s}}(a) \leqq 0\right)$;

e) $\sum_{i=1}^{k} \bar{\lambda}_{i}\left(\rho_{f_{i}}+\bar{v}_{i} \rho_{q_{i}}\right)+\sum_{j \in J(\bar{x})} \bar{\mu}_{j} \rho_{g_{j}}+\sum_{s \in S^{+}(\bar{x})} \bar{\omega}_{s} \rho_{h_{s}}^{+}-\sum_{s \in S^{-}(\bar{x})} \bar{\omega}_{s} \rho_{h_{s}}^{-} \geqq 0$.

Then $\bar{x}$ is a Pareto solution of the considered nonsmooth multiobjective fractional programming problem (MFP).

\section{Parametric duality}

Now, for the considered nonsmooth fractional multiobjective programming problem (MFP), we consider a vector dual problem $(\mathrm{SD})$ in the sense of Schaible as follows:

$$
\begin{gathered}
\text { Maximize } v=\left(v_{1}, \ldots, v_{k}\right) \\
\text { subject to } 0 \in \sum_{i=1}^{k} \lambda_{i} \partial\left(f_{i}(y)-v_{i} q_{i}(y)\right)+\sum_{j=1}^{m} \mu_{j} \partial g_{j}(y)+\sum_{s=1}^{p} \omega_{s} \partial h_{s}(y) \\
f_{i}(y)-v_{i} q_{i}(y) \geqq 0, i=1, \ldots, k, \quad(\mathrm{SD}) \\
\mu_{j} g_{j}(u) \geqq 0, j=1, \ldots, m, \\
\omega_{s} h_{s}(y) \geqq 0, \quad s=1, \ldots, p, \\
y \in X, \lambda_{i} \geqq 0, i=1, \ldots, k, \sum_{i=1}^{k} \lambda_{i}=1, \mu_{j} \geqq 0, j=1, \ldots, m .
\end{gathered}
$$

Let $\Gamma$ denote the set of all feasible solutions in the problem (SD), that is, the set of $(y, v, \lambda, \mu, \omega)$ verifying the constraints (35)-(39). Further, we denote by $\Omega$ the set $\Omega=\{y \in X:(y, v, \lambda, \mu, \omega) \in \Gamma\}$ and, for $y \in \Omega$, $J(y)=\left\{j \in J: g_{j}(y)=0\right\}$. 
Theorem 16 (Weak duality). Let $x$ and $(y, v, \lambda, \mu, \omega)$ be feasible solutions of the problems (MFP) and (SD), respectively. Further, assume that one of the following two sets of hypotheses is satisfied:

A) a) for each $i=1, \ldots, k, f_{i}(\cdot)-v_{i} q_{i}(\cdot)$ is $\left(b_{i}, \Psi_{i}, \Phi, \rho_{i}\right)$-univex at $y$ on $D \cup \Omega$ and $a<0 \Longrightarrow \Psi_{i}(a)<0$;

b) for each $j \in J(y), g_{j}(\cdot)$ is $\left(b_{g_{j}}, \Psi_{g_{j}}, \Phi, \rho_{g_{j}}\right)$-univex at $y$ on $D \cup \Omega$ and $a \leqq 0 \Longrightarrow \Psi_{g_{j}}(a) \leqq 0$;

c) for each $s \in S^{+}(y), h_{s}(\cdot)$ is $\left(b_{h_{s}}, \Psi_{h_{s}}, \Phi, \rho_{h_{s}}^{+}\right)$-univex at y on $D \cup \Omega, \Psi_{h_{s}}$ is a positively homogeneous function and $a \leqq 0 \Longrightarrow \Psi_{h_{s}}(a) \leqq 0$;

d) for each $s \in S^{-}(y),-h_{s}(\cdot)$ is $\left(b_{h_{s}}, \Psi_{h_{s}}, \Phi, \rho_{h_{s}}^{-}\right)$-univex at $y$ on $D \cup \Omega, \Psi_{h_{s}}$ is a positively homogeneous function and $a \leqq 0 \Longrightarrow \Psi_{h_{s}}(a) \leqq 0$;

e) $\sum_{i=1}^{k} \lambda_{i} \rho_{i}+\sum_{j \in J(y)} \mu_{j} \rho_{g_{j}}+\sum_{s \in S^{+}(y)} \omega_{s} \rho_{h_{s}}^{+}-\sum_{s \in S^{-}(y)} \omega_{s} \rho_{h_{s}}^{-} \geqq 0$.

B) a) for each $i=1, \ldots, k, f_{i}(\cdot)$ is $\left(b_{i}, \Psi_{i}, \Phi, \rho_{f_{i}}\right)$-univex at $y$ on $D \cup \Omega$ and $-q_{i}(\cdot)$ are $\left(b_{i}, \Psi_{i}, \Phi, \rho_{q_{i}}\right)$ univex at $y$ on $D \cup \Omega, \Psi_{i}$ is a superlinear function and $a<0 \Longrightarrow \Psi_{i}(a)<0$;

b) for each $j \in J(y), g_{j}(\cdot)$ is $\left(b_{g_{j}}, \Psi_{g_{j}}, \Phi, \rho_{g_{j}}\right)$-univex at $y$ on $D \cup \Omega$ and $a \leqq 0 \Longrightarrow \Psi_{g_{j}}(a) \leqq 0$;

c) for each $s \in S^{+}(y), h_{s}(\cdot)$ is $\left(b_{h_{s}}, \Psi_{h_{s}}, \Phi, \rho_{h_{s}}^{+}\right)$-univex at $y$ on $D \cup \Omega, \Psi_{h_{s}}$ is a positively homogeneous function and $a \leqq 0 \Longrightarrow \Psi_{h_{s}}(a) \leqq 0 ;$

d) for each $s \in S^{-}(y),-h_{s}(\cdot)$ is $\left(b_{h_{s}}, \Psi_{h_{s}}, \Phi, \rho_{h_{s}}^{-}\right)$-univex at $y$ on $D \cup \Omega, \Psi_{h_{s}}$ is a positively homogeneous function and $a \leqq 0 \Longrightarrow \Psi_{h_{s}}(a) \leqq 0$;

e) $\sum_{i=1}^{k} \lambda_{i}\left(\rho_{f_{i}}+v_{i} \rho_{q_{i}}\right)+\sum_{j \in J(y)} \mu_{j} \rho_{g_{j}}+\sum_{s \in S^{+}(y)} \omega_{s} \rho_{h_{s}}^{+}-\sum_{s \in S^{-}(y)} \omega_{s} \rho_{h_{s}}^{-} \geqq 0$.

Then

$$
\varphi(x) \nless v .
$$

Proof We prove this theorem under hypothesis A).

By means of contradiction, suppose that there exist $x \in D$ and $(y, v, \lambda, \mu, \omega) \in \Gamma$ such that

$$
\varphi(x)<v
$$

Thus, by the definition of the function $\varphi$, (40) implies

$$
f_{i}(x)-v_{i} q_{i}(x)<0, i=1, \ldots, k .
$$

Then the constraint (36) gives

$$
f_{i}(x)-v_{i} q_{i}(x)<f_{i}(y)-v_{i} q_{i}(y), i=1, \ldots, k .
$$

By hypothesis a), (41) yields

$$
b_{i}(x, y) \Psi_{i}\left(f_{i}(x)-v_{i} q_{i}(x)-\left(f_{i}(y)-v_{i} q_{i}(y)\right)\right)<0, i=1, \ldots, k .
$$

Hence, by Definition 7, inequalities (42) imply that the following inequalities

$$
\Phi\left(x, y,\left(\xi_{i}, \rho_{i}\right)\right)<0, i=1, \ldots, k
$$


hold for each $\xi_{i} \in \partial\left(f_{i}(y)-v_{i} q_{i}(y)\right), i=1, \ldots, k$. Since $\lambda_{i} \geqq 0, \sum_{i=1}^{k} \lambda_{i}=1$, inequalities (43) imply

$$
\sum_{i=1}^{k} \lambda_{i} \Phi\left(x, y,\left(\xi_{i}, \rho_{i}\right)\right)<0 .
$$

By $x \in D$ and $(y, v, \lambda, \mu, \omega) \in \Gamma$, we have that $g_{j}(x) \leqq g_{j}(u), j \in J(y)$. Using hypothesis b), by (44) and Definition 7 , we have

$$
b_{g_{j}}(x, y) \Psi_{g_{j}}\left(g_{j}(x)-g_{j}(y)\right) \leqq 0, j \in J(y) .
$$

Using Definition 7 again, by (45), we obtain that the inequalities

$$
\Phi\left(x, y,\left(\zeta_{j}, \rho_{g_{j}}\right)\right) \leq 0, j \in J(y)
$$

hold for each $\zeta_{j} \in \partial g_{j}(y), j \in J(y)$. Since $\mu_{j} \geqq 0, j \in J$, the above inequalities yield

$$
\sum_{j \in J(y)} \mu_{j} \Phi\left(x, y,\left(\zeta_{j}, \rho_{g_{j}}\right)\right) \leqq 0
$$

Since, by hypotheses c) and d), each function $h_{s}, s \in S^{+}(y)$, is locally Lipschitz $\left(b_{h_{s}}, \Psi_{h_{s}}, \Phi, \rho_{h_{s}}^{+}\right)$-univex at $y$ on $D \cup \Omega$ and each function $-h_{s}, s \in S^{-}(y)$, is locally Lipschitz $\left(b_{h_{s}}, \Psi_{h_{s}}, \Phi, \rho_{h_{s}}^{-}\right)$-univex at $y$ on $D \cup \Omega$, by Definition 7, the following inequalities

$$
\begin{gathered}
b_{h_{s}}(x, y) \Psi_{h_{s}}\left(h_{s}(x)-h_{s}(y)\right) \geqq \Phi\left(x, y,\left(\varsigma_{s}, \rho_{h_{s}}^{+}\right)\right), \quad s \in S^{+}(y), \\
b_{h_{s}}(x, y) \Psi_{h_{s}}\left(-h_{s}(x)+h_{s}(y)\right) \geqq \Phi\left(x, y,\left(-\varsigma_{s}, \rho_{h_{s}}^{-}\right)\right), \quad s \in S^{-}(y)
\end{gathered}
$$

hold for any $\varsigma_{s} \in \partial h_{s}(y), s \in S^{+}(y)$ and $-\varsigma_{s} \in \partial\left(-h_{s}\right)(y), s \in S^{-}(y)$, respectively. Multiplying each inequality (47) by the corresponding Lagrange multiplier $\omega_{s}>0, s \in S^{+}(y)$, and each inequality (48) by $-\omega_{s}>0, s \in S^{-}(y)$, respectively, we obtain

$$
\begin{gathered}
b_{h_{s}}(x, y) \omega_{s} \Psi_{h_{s}}\left(h_{s}(x)-h_{s}(y)\right) \geqq \omega_{s} \Phi\left(x, y,\left(\varsigma_{s}, \rho_{h_{s}}^{+}\right)\right), \quad s \in S^{+}(y), \\
-b_{h_{s}}(x, y) \omega_{s} \Psi_{h_{s}}\left(-h_{s}(x)+h_{s}(y)\right) \geqq-\omega_{s} \Phi\left(x, y,\left(-\varsigma_{s}, \rho_{h_{s}}^{-}\right)\right), \quad s \in S^{-}(y)
\end{gathered}
$$

Since each $\Psi_{h_{s}}, s \in S^{+}(y) \cup S^{-}(y)$ is a positively homogeneous function (see Definition 6 ii)), inequalities (49) and (50) yield, respectively,

$$
\begin{gathered}
b_{h_{s}}(x, y) \Psi_{h_{s}}\left(\omega_{s} h_{s}(x)-\omega_{s} h_{s}(y)\right) \geqq \omega_{s} \Phi\left(x, y,\left(\varsigma_{s}, \rho_{h_{s}}^{+}\right)\right), \quad s \in S^{+}(y), \\
b_{h_{s}}(x, y) \omega_{s} \Psi_{h_{s}}\left(\omega_{s} h_{s}(x)-\omega_{s} h_{s}(y)\right) \geqq-\omega_{s} \Phi\left(x, y,\left(-\varsigma_{s}, \rho_{h_{s}}^{-}\right)\right), \quad s \in S^{-}(y) .
\end{gathered}
$$

By $x \in D$ and $(y, v, \lambda, \mu, \omega) \in \Omega$, we have

$$
\omega_{s} h_{s}(x)-\omega_{s} h_{s}(y) \leqq 0, \quad s \in S .
$$


By hypotheses c) and d), (53) gives

$$
b_{h_{s}}(x, y) \Psi_{h_{s}}\left(\omega_{s} h_{s}(x)-\omega_{s} h_{s}(y)\right) \leqq 0, s \in S .
$$

Combining (51), (52), and (54) we get, respectively,

$$
\begin{gathered}
\omega_{s} \Phi\left(x, y,\left(\varsigma_{s}, \rho_{h_{s}}^{+}\right)\right) \leqq 0, \quad s \in S^{+}(y), \\
-\omega_{s} \Phi\left(x, y,\left(-\varsigma_{s}, \rho_{h_{s}}^{-}\right)\right) \leqq 0, \quad s \in S^{-}(y) .
\end{gathered}
$$

Thus, (55) and (56) yield that the inequalities

$$
\begin{gathered}
\sum_{s \in S^{+}(y)} \omega_{s} \Phi\left(x, y,\left(\varsigma_{s}, \rho_{h_{s}}^{+}\right)\right) \leqq 0, \\
\sum_{s \in S^{-}(y)}\left(-\omega_{s}\right) \Phi\left(x, y,\left(-\varsigma_{s}, \rho_{h_{s}}^{-}\right)\right) \leqq 0
\end{gathered}
$$

hold for any $\varsigma_{s} \in \partial h_{s}(y), s \in S^{+}(y)$ and $-\varsigma_{s} \in \partial\left(-h_{s}\right)(y), s \in S^{-}(y)$, respectively. Combining (44), (46), (57), and (58), we get

$$
\begin{gathered}
\sum_{i=1}^{k} \lambda_{i} \Phi\left(x, y,\left(\xi_{i}, \rho_{i}\right)\right)+\sum_{j \in J(y)} \mu_{j} \Phi\left(x, y,\left(\zeta_{j}, \rho_{g_{j}}\right)\right)+ \\
\sum_{s \in S^{+}(y)} \omega_{s} \Phi\left(x, y,\left(\varsigma_{s}, \rho_{h_{s}}^{+}\right)\right)+\sum_{s \in S^{-}(y)}\left(-\omega_{s}\right) \Phi\left(x, y,\left(-\varsigma_{s}, \rho_{h_{s}}^{-}\right)\right)<0 .
\end{gathered}
$$

Let us denote by

$$
\begin{gathered}
A=\sum_{i=1}^{k} \lambda_{i}+\sum_{j=1}^{m} \mu_{j}+\sum_{s=1}^{p} \omega_{s}, \\
\alpha_{i}=\frac{\lambda_{i}}{A}, i \in I, \\
\beta_{j}=\frac{\mu_{j}}{A}, j \in J, \\
\gamma_{s}^{+}=\frac{\omega_{s}}{A}, s \in S^{+}(y), \\
\bar{\gamma}_{s}^{-}=\frac{-\omega_{s}}{A}, s \in S^{-}(y) .
\end{gathered}
$$

Then, by $x \in D$ and $(y, v, \lambda, \mu, \omega) \in \Gamma$, we have that $\alpha:=\left(\alpha_{1}, \ldots, \alpha_{k}\right) \geq 0,0 \leqq \alpha_{i} \leqq 1, i \in I$, but at least one $\alpha_{i}>0, \beta_{j}=\left(\beta_{1}, \ldots, \beta_{m}\right) \geqq 0,0 \leqq \beta_{j} \leqq 1, j \in J, 0 \leqq \gamma_{s}^{+} \leqq 1, s \in S^{+}(y), 0 \leqq \gamma_{s}^{-} \leqq 1, s \in S^{-}(y), A>0$, and, moreover, $\sum_{i=1}^{k} \alpha_{i}+\sum_{j=1}^{m} \beta_{j}+\sum_{s \in S^{+}(y)} \gamma_{s}^{+}+\sum_{s \in S^{-}(y)} \gamma_{s}^{-}=1$. Using (60)-(64) in (59), we get

$$
\sum_{i=1}^{k} \alpha_{i} \Phi\left(x, y,\left(\xi_{i}, \rho_{i}\right)\right)+\sum_{j \in J(y)} \beta_{j} \Phi\left(x, y,\left(\zeta_{j}, \rho_{g_{j}}\right)\right)+
$$




$$
\sum_{s \in S^{+}(y)} \gamma_{s}^{+} \Phi\left(x, y,\left(\varsigma_{s}, \rho_{h_{s}}^{+}\right)\right)+\sum_{s \in S^{-}(y)} \gamma_{s}^{-} \Phi\left(x, y,\left(-\varsigma_{s}, \rho_{h_{s}}^{-}\right)\right)<0 .
$$

By Definition 7 , we have that $\Phi(x, y, \cdot)$ is a convex function on $R^{n+1}$. Thus, by the definition of convexity, the above inequality gives

$$
\begin{gathered}
\Phi\left(x, y,\left(\sum_{i=1}^{k} \alpha_{i} \xi_{i}+\sum_{j \in J(y)} \beta_{j} \zeta_{j}+\sum_{s \in S^{+}(y)} \gamma_{s}^{+} \varsigma_{s}+\sum_{s \in S^{-}(y)} \gamma_{s}^{-}\left(-\varsigma_{s}\right),\right.\right. \\
\left.\left.\sum_{i=1}^{k} \alpha_{i} \rho_{i}+\sum_{j \in J(y)} \beta_{j} \rho_{g_{j}}+\sum_{s \in S^{+}(y)} \gamma_{s}^{+} \rho_{h_{s}}^{+}+\sum_{s \in S^{-}(y)} \gamma_{s}^{-} \rho_{h_{s}}^{-}\right)\right)<0 .
\end{gathered}
$$

Using (60)-(64) in (65), we obtain

$$
\begin{gathered}
\Phi\left(x, y, \frac{1}{A}\left(\sum_{i=1}^{k} \lambda_{i} \xi_{i}+\sum_{j \in J(y)} \mu_{j} \zeta_{j}+\sum_{s \in S^{+}(y) \cup S^{-}(y)} \omega_{s} \varsigma_{s},\right.\right. \\
\left.\left.\sum_{i=1}^{k} \lambda_{i} \rho_{i}+\sum_{j \in J(y)} \mu_{j} \rho_{g_{j}}+\sum_{s \in S^{+}(y)} \omega_{s} \rho_{h_{s}}^{+}-\sum_{s \in S^{-}(y)} \omega_{s} \rho_{h_{s}}^{-}\right)\right)<0 .
\end{gathered}
$$

If we take the Lagrange multipliers $\mu_{j}=0, j \notin J(y), \omega_{s}=0, s \notin S^{+}(y) \cup S^{-}(y)$ in (66), then we have that the following inequality

$$
\begin{gathered}
\Phi\left(x, y, \frac{1}{A}\left(\sum_{i=1}^{k} \lambda_{i} \xi_{i}+\sum_{j=1}^{m} \mu_{j} \zeta_{j}+\sum_{s=1}^{p} \omega_{s} \varsigma_{s}\right.\right. \\
\left.\left.\sum_{i=1}^{k} \lambda_{i} \rho_{i}+\sum_{j \in J(y)} \mu_{j} \rho_{g_{j}}+\sum_{s \in S^{+}(y)} \omega_{s} \rho_{h_{s}}^{+}-\sum_{s \in S^{-}(y)} \omega_{s} \rho_{h_{s}}^{-}\right)\right)<0
\end{gathered}
$$

holds for any $\xi_{i} \in \partial\left(f_{i}-v_{i} q_{i}\right)(y), i \in I, \zeta_{j} \in \partial g_{j}(y), j \in J, \varsigma_{s} \in \partial h_{s}(y), s \in S$. Hence, by the first constraint in the problem (SD), we have

$$
\Phi\left(x, y, \frac{1}{A}\left(0, \sum_{i=1}^{k} \lambda_{i} \rho_{i}+\sum_{j \in J(y)} \mu_{j} \rho_{g_{j}}+\sum_{s \in S^{+}(y)} \omega_{s} \rho_{h_{s}}^{+}-\sum_{s \in S^{-}(y)} \omega_{s} \rho_{h_{s}}^{-}\right)\right)<0
$$

By hypothesis e), we have that $\sum_{i=1}^{k} \lambda_{i} \rho_{f_{i}}+\sum_{j \in J(y)} \mu_{j} \rho_{g_{j}}+\sum_{s \in S^{+}(y)} \omega_{s} \rho_{h_{s}}^{+}-\sum_{s \in S^{-}(y)} \omega_{s} \rho_{h_{s}}^{-} \geqq 0$. Thus, by Definition 7 , the following inequality

$$
\Phi\left(x, y, \frac{1}{A}\left(0, \sum_{i=1}^{k} \lambda_{i} \rho_{i}+\sum_{j \in J(y)} \mu_{j} \rho_{g_{j}}+\sum_{s \in S^{+}(y)} \omega_{s} \rho_{h_{s}}^{+}-\sum_{s \in S^{-}(y)} \omega_{s} \rho_{h_{s}}^{-}\right)\right) \geqq 0
$$

holds, contradicting (67). Thus, the proof of this theorem under hypothesis A) is completed. 
Proof of the theorem under hypothesis B) is similar to that under hypothesis A) and, therefore, it has been omitted from the paper.

Under slightly stronger $(b, \Psi, \Phi, \rho)$-univexity assumptions imposed on the objective function, the following stronger result is true:

Theorem 17 (Weak duality). Let $x$ and $(y, v, \lambda, \mu, \omega)$ be feasible solutions for the problems (MFP) and (SD), respectively. Further, assume that one of the following two sets of hypotheses is satisfied:

A) a) for each $i=1, \ldots, k, f_{i}(\cdot)-v_{i} q_{i}(\cdot)$ is strictly $\left(b_{i}, \Psi_{i}, \Phi, \rho_{i}\right)$-univex at y on $D \cup \Omega$ and a $0 \Longrightarrow$ $\Psi_{i}(a) \leqq 0 ;$

b) for each $j \in J(y), g_{j}(\cdot)$ is $\left(b_{g_{j}}, \Psi_{g_{j}}, \Phi, \rho_{g_{j}}\right)$-univex at $y$ on $D \cup \Omega$ and $a \leqq 0 \Longrightarrow \Psi_{g_{j}}(a) \leqq 0$;

c) for each $s \in S^{+}(y), h_{s}(\cdot)$ is $\left(b_{h_{s}}, \Psi_{h_{s}}, \Phi, \rho_{h_{s}}^{+}\right)$-univex at $y$ on $D \cup \Omega, \Psi_{h_{s}}$ is a positively homogeneous function and $a \leqq 0 \Longrightarrow \Psi_{h_{s}}(a) \leqq 0$;

d) for each $s \in S^{-}(y),-h_{s}(\cdot)$ is $\left(b_{h_{s}}, \Psi_{h_{s}}, \Phi, \rho_{h_{s}}^{-}\right)$-univex at $y$ on $D \cup \Omega, \Psi_{h_{s}}$ is a positively homogeneous function and $a \leqq 0 \Longrightarrow \Psi_{h_{s}}(a) \leqq 0$;

e) $\sum_{i=1}^{k} \lambda_{i} \rho_{i}+\sum_{j \in J(y)} \mu_{j} \rho_{g_{j}}+\sum_{s \in S^{+}(y)} \omega_{s} \rho_{h_{s}}^{+}-\sum_{s \in S^{-}(y)} \omega_{s} \rho_{h_{s}}^{-} \geqq 0$.

B) a) for each $i=1, \ldots, k, f_{i}(\cdot)$ is strictly $\left(b_{i}, \Psi_{i}, \Phi, \rho_{f_{i}}\right)$-univex at $y$ on $D \cup \Omega$ and $-q_{i}(\cdot)$ is strictly $\left(b_{i}, \Psi_{i}, \Phi, \rho_{q_{i}}\right)$-univex at $y$ on $D \cup \Omega, \Psi_{i}$ is a superlinear function and $a \leqq 0 \Longrightarrow \Psi_{i}(a) \leqq 0$;

b) for each $j \in J(y), g_{j}(\cdot)$ is $\left(b_{g_{j}}, \Psi_{g_{j}}, \Phi, \rho_{g_{j}}\right)$-univex at $y$ on $D \cup \Omega$ and $a \leqq 0 \Longrightarrow \Psi_{g_{j}}(a) \leqq 0$;

c) for each $s \in S^{+}(y), h_{s}(\cdot)$ is $\left(b_{h_{s}}, \Psi_{h_{s}}, \Phi, \rho_{h_{s}}^{+}\right)$-univex at y on $D \cup \Omega, \Psi_{h_{s}}$ is a positively homogeneous function and $a \leqq 0 \Longrightarrow \Psi_{h_{s}}(a) \leqq 0$;

d) for each $s \in S^{-}(y),-h_{s}(\cdot)$ is $\left(b_{h_{s}}, \Psi_{h_{s}}, \Phi, \rho_{h_{s}}^{-}\right)$-univex at $y$ on $D \cup \Omega, \Psi_{h_{s}}$ is a positively homogeneous function and $a \leqq 0 \Longrightarrow \Psi_{h_{s}}(a) \leqq 0$;

e) $\sum_{i=1}^{k} \lambda_{i}\left(\rho_{f_{i}}+v_{i} \rho_{q_{i}}\right)+\sum_{j \in J(y)} \mu_{j} \rho_{g_{j}}+\sum_{s \in S^{+}(y)} \omega_{s} \rho_{h_{s}}^{+}-\sum_{s \in S^{-}(y)} \omega_{s} \rho_{h_{s}}^{-} \geqq 0$.

Then

$$
\varphi(x) \not \leq .
$$

Theorem 18 (Strong duality). Let $\bar{x}$ be a weak Pareto solution (Pareto solution) of the considered multiobjective fractional programming problem (MFP) and the generalized Slater constraint qualification be satisfied at $\bar{x}$. Then there exist Lagrange multipliers $\bar{\lambda} \in R^{k}, \bar{\mu} \in R^{m}, \bar{\omega} \in R^{p}$ and, moreover, $\bar{\nu} \in R^{k}$ such that $(\bar{x}, \bar{v}, \bar{\lambda}, \bar{\mu}, \bar{\omega})$ is feasible solution in (SD). If also all hypotheses of Theorem 16 (Theorem 17) hold, then $(\bar{x}, \bar{v}, \bar{\lambda}, \bar{\mu}, \bar{\omega})$ is a weakly efficient solution (efficient solution) of a maximum type for the problem (SD) and the corresponding optimal values of (MFP) and (SD) are the same.

Proof By assumption, $\bar{x} \in D$ is a (weak) Pareto solution of the problem (MFP) with $\bar{v}=\varphi(\bar{x})$ and the generalized Slater constraint qualification is satisfied at $\bar{x}$. Hence, by Theorem 12 , there exist $\bar{\lambda} \in R^{k}, \bar{\mu} \in R^{m}$, and $\bar{\omega} \in R^{p}$ such that $(\bar{x}, \bar{v}, \bar{\lambda}, \bar{\mu}, \bar{\omega})$ is feasible in the problem (SD). Since

$$
\bar{v}=\varphi(\bar{x})
$$


ANTCZAK and VERMA/Turk J Math

using the weak duality theorem (Theorem 16 or Theorem 17$)$, we conclude that $(\bar{x}, \bar{v}, \bar{\lambda}, \bar{\mu}, \bar{\omega})$ is a weakly efficient solution (efficient solution) of a maximum type for the problem (SD). Hence, the corresponding optimal values in problems $(\mathrm{MFP})$ and $(\mathrm{SD})$ are the same. This completes the proof of this theorem.

Theorem 19 (Converse Duality). Let $(\bar{x}, \bar{v}, \bar{\lambda}, \bar{\mu}, \bar{\omega})$ be a weakly efficient solution (efficient solution) of a maximum type for the Schaible dual problem (SD) such that $\bar{x} \in D$. Further, assume that one of the following two sets of hypotheses is satisfied:

A) a) for each $i=1, \ldots, k, f_{i}(\cdot)-\bar{v}_{i} q_{i}(\cdot)$ is (strictly) $\left(b_{i}, \Psi_{i}, \Phi, \rho_{i}\right)$-univex at $\bar{x}$ on $D \cup \Omega, \Psi_{i}$ is a superlinear function and $a<0 \Longrightarrow \Psi_{i}(a)<0 \quad\left(a \leqq 0 \Longrightarrow \Psi_{h_{j}}(a) \leqq 0\right)$;

b) for each $j \in J(\bar{x}), g_{j}(\cdot)$ is $\left(b_{g_{j}}, \Psi_{g_{j}}, \Phi, \rho_{g_{j}}\right)$-univex at $\bar{x}$ on $D \cup \Omega$ and $a \leqq 0 \Longrightarrow \Psi_{g_{j}}(a) \leqq 0$;

c) for each $s \in S^{+}(\bar{x}), h_{s}(\cdot)$ is $\left(b_{h_{s}}, \Psi_{h_{s}}, \Phi, \rho_{h_{s}}^{+}\right)$-univex at $\bar{x}$ on $D \cup \Omega, \Psi_{h_{s}}$ is a positively homogeneous function and $a \leqq 0 \Longrightarrow \Psi_{h_{s}}(a) \leqq 0$;

d) for each $s \in S^{-}(\bar{x}),-h_{s}(\cdot)$ is $\left(b_{h_{s}}, \Psi_{h_{s}}, \Phi, \rho_{h_{s}}^{-}\right)$-univex at $\bar{x}$ on $D \cup \Omega, \Psi_{h_{s}}$ is a positively homogeneous function and $a \leqq 0 \Longrightarrow \Psi_{h_{s}}(a) \leqq 0$;

e) $\sum_{i=1}^{k} \bar{\lambda}_{i} \rho_{i}+\sum_{j \in J(\bar{x})} \bar{\mu}_{j} \rho_{g_{j}}+\sum_{s \in S^{+}(\bar{x})} \bar{\omega}_{s} \rho_{h_{s}}^{+}-\sum_{s \in S^{-}(\bar{x})} \bar{\omega}_{s} \rho_{h_{s}}^{-} \geqq 0$.

B) a) for each $i=1, \ldots, k, f_{i}(\cdot)$ is (strictly) $\left(b_{i}, \Psi_{i}, \Phi, \rho_{f_{i}}\right)$-univex at $\bar{x}$ on $D \cup \Omega$ and $-q_{i}(\cdot)$ is (strictly) $\left(b_{i}, \Psi_{i}, \Phi, \rho_{q_{i}}\right)$-univex at $\bar{x}$ on $D \cup \Omega, \Psi_{i}$ is a superlinear function and $a<0 \Longrightarrow \Psi_{i}(a)<0$ $\left(a \leqq 0 \Longrightarrow \Psi_{h_{j}}(a) \leqq 0\right)$;

b) for each $j \in J(\bar{x}), g_{j}(\cdot)$ is $\left(b_{g_{j}}, \Psi_{g_{j}}, \Phi, \rho_{g_{j}}\right)$-univex at $\bar{x}$ on $D \cup \Omega$ and $a \leqq 0 \Longrightarrow \Psi_{g_{j}}(a) \leqq 0$;

c) for each $s \in S^{+}(\bar{x}), h_{s}(\cdot)$ is $\left(b_{h_{s}}, \Psi_{h_{s}}, \Phi, \rho_{h_{s}}^{+}\right)$-univex at $\bar{x}$ on $D \cup \Omega, \Psi_{h_{s}}$ is a positively homogeneous function and $a \leqq 0 \Longrightarrow \Psi_{h_{s}}(a) \leqq 0$;

d) for each $s \in S^{-}(\bar{x}),-h_{s}(\cdot)$ is $\left(b_{h_{s}}, \Psi_{h_{s}}, \Phi, \rho_{h_{s}}^{-}\right)$-univex at $\bar{x}$ on $D \cup \Omega, \Psi_{h_{s}}$ is a positively homogeneous function and $a \leqq 0 \Longrightarrow \Psi_{h_{s}}(a) \leqq 0$;

e) $\sum_{i=1}^{k} \bar{\lambda}_{i}\left(\rho_{f_{i}}+\bar{v}_{i} \rho_{q_{i}}\right)+\sum_{j \in J(\bar{x})} \bar{\mu}_{j} \rho_{g_{j}}+\sum_{s \in S^{+}(\bar{x})} \bar{\omega}_{s} \rho_{h_{s}}^{+}-\sum_{s \in S^{-}(\bar{x})} \bar{\omega}_{s} \rho_{h_{s}}^{-} \geqq 0$.

Then $\bar{x}$ is a weak Pareto solution (Pareto solution) of the considered nonsmooth multiobjective fractional programming problem (MFP).

Proof Proof of this theorem follows directly from weak duality (Theorem 16).

Theorem 20 (Strict Converse Duality). Let $\bar{x}$ be a weak Pareto solution of the considered nonsmooth multiobjective fractional programming problem (MFP), $(\bar{y}, \bar{v}, \bar{\lambda}, \bar{\mu}, \bar{\omega})$ be a weakly efficient solution of a maximum type for the Schaible dual problem (SD) and the generalized Slater constraint qualification be satisfied at $\bar{x}$. Assume, furthermore, that one of the sets of hypotheses is fulfilled:

A) $\quad$ a) for each $i=1, \ldots, k, f_{i}(\cdot)-\bar{v}_{i} q_{i}(\cdot)$ is strictly $\left(b_{i}, \Psi_{i}, \Phi, \rho_{i}\right)$-univex at $\bar{y}$ on $D \cup \Omega, a \leqq 0 \Longrightarrow \Psi(a) \leqq$ 0 
b) for each $j \in J(\bar{y}), g_{j}(\cdot)$ is $\left(b_{g_{j}}, \Psi_{g_{j}}, \Phi, \rho_{g_{j}}\right)$-univex at $\bar{y}$ on $D \cup \Omega$ and $a \leqq 0 \Longrightarrow \Psi_{g_{j}}(a) \leqq 0$;

c) for each $s \in S^{+}(\bar{y}), h_{s}(\cdot)$ is $\left(b_{h_{s}}, \Psi_{h_{s}}, \Phi, \rho_{h_{s}}^{+}\right)$-univex at $\bar{y}$ on $D \cup \Omega, \Psi_{h_{s}}$ is a positively homogeneous function and $a \leqq 0 \Longrightarrow \Psi_{h_{j}}(a) \leqq 0 ;$

d) for each $s \in S^{-}(\bar{y}),-h_{s}(\cdot)$ is $\left(b_{h_{s}}, \Psi_{h_{s}}, \Phi, \rho_{h_{s}}^{-}\right)$-univex at $\bar{y}$ on $D \cup \Omega, \Psi_{h_{s}}$ is a positively homogeneous function and $a \leqq 0 \Longrightarrow \Psi_{h_{j}}(a) \leqq 0$;

e) $\sum_{i=1}^{k} \bar{\lambda}_{i} \rho_{f_{i}}+\sum_{j \in J(\bar{y})} \bar{\mu}_{j} \rho_{g_{j}}+\sum_{s \in S^{+}(\bar{y})} \bar{\omega}_{s} \rho_{h_{s}}^{+}-\sum_{s \in S^{-}(\bar{y})} \bar{\omega}_{s} \rho_{h_{s}}^{-} \geqq 0$.

B) a) for each $i=1, \ldots, k, f_{i}(\cdot)$ and $-q_{i}(\cdot)$ are strictly $\left(b_{i}, \Psi_{i}, \Phi, \rho_{i}\right)$-univex at $\bar{x}$ on $D \cup \Omega, \Psi_{i}$ is a superlinear function and $a \leqq 0 \Longrightarrow \Psi_{i}(a) \leqq 0$;

b) for each $j \in J(\bar{y}), g_{j}(\cdot)$ is $\left(b_{g_{j}}, \Psi_{g_{j}}, \Phi, \rho_{g_{j}}\right)$-univex at $\bar{y}$ on $D \cup \Omega$ and $a \leqq 0 \Longrightarrow \Psi_{g_{j}}(a) \leqq 0$;

c) for each $s \in S^{+}(\bar{y}), h_{s}(\cdot)$ is $\left(b_{h_{s}}, \Psi_{h_{s}}, \Phi, \rho_{h_{s}}^{+}\right)$-univex at $\bar{y}$ on $D \cup \Omega, \Psi_{h_{s}}$ is a positively homogeneous function and $a \leqq 0 \Longrightarrow \Psi_{h_{j}}(a) \leqq 0 ;$

d) for each $s \in S^{-}(\bar{y}),-h_{s}(\cdot)$ is $\left(b_{h_{s}}, \Psi_{h_{s}}, \Phi, \rho_{h_{s}}^{-}\right)$-univex at $\bar{y}$ on $D \cup \Omega, \Psi_{h_{s}}$ is a positively homogeneous function and $a \leqq 0 \Longrightarrow \Psi_{h_{j}}(a) \leqq 0$;

e) $\sum_{i=1}^{k} \bar{\lambda}_{i}\left(\rho_{f_{i}}+\bar{v}_{i} \rho_{q_{i}}\right)+\sum_{j \in J(\bar{y})} \bar{\mu}_{j} \rho_{g_{j}}+\sum_{s \in S^{+}(\bar{y})} \bar{\omega}_{s} \rho_{h_{s}}^{+}-\sum_{s \in S^{-}(\bar{y})} \bar{\omega}_{s} \rho_{h_{s}}^{-} \geqq 0$.

Then $\bar{x}=\bar{y}$.

Proof We now prove the theorem under hypothesis A). Suppose that $\bar{x} \neq \bar{y}$ and exhibits a contradiction. By $(\bar{y}, \bar{v}, \bar{\lambda}, \bar{\mu}, \bar{\omega}) \in \Gamma$, it follows that there exist $\xi_{i} \in \partial\left(f_{i}-\bar{v}_{i} q_{i}\right)(\bar{y}), i \in I, \zeta_{j} \in \partial g_{j}(\bar{y}), j \in J, \varsigma_{s} \in \partial h_{s}(\bar{y})$, $s \in S$ such that

$$
0 \in \sum_{i=1}^{k} \bar{\lambda}_{i} \xi_{i}+\sum_{j=1}^{m} \bar{\mu}_{j} \zeta_{j}+\sum_{s=1}^{p} \bar{\omega}_{s} \varsigma_{s} .
$$

Also by $(\bar{y}, \bar{v}, \bar{\lambda}, \bar{\mu}, \bar{\omega}) \in \Gamma$, we have

$$
f_{i}(\bar{y})-\bar{v}_{i} q_{i}(\bar{y}) \geqq 0, i=1, \ldots, k .
$$

By the strong duality (Theorem 18), it follows that

$$
\bar{v}=\varphi(\bar{x}) .
$$

This implies that

$$
f_{i}(\bar{x})-\bar{v}_{i} q_{i}(\bar{x})=0, i=1, \ldots, k .
$$

Combining (69) and (70), we obtain

$$
f_{i}(\bar{x})-\bar{v}_{i} q_{i}(\bar{x}) \leqq f_{i}(\bar{y})-\bar{v}_{i} q_{i}(\bar{y}), i=1, \ldots, k .
$$

By hypothesis a) and $b_{i}(\bar{x}, \bar{y})>0, i=1, \ldots, k$, we have

$$
b_{i}(\bar{x}, \bar{y}) \Psi_{i}\left(f_{i}(\bar{x})-\bar{v}_{i} q_{i}(\bar{x})-\left(f_{i}(\bar{y})-\bar{v}_{i} q_{i}(\bar{y})\right)\right) \leqq 0, i=1, \ldots, k .
$$


Using hypothesis a) again, by Definition 7, the following inequality

$$
\sum_{i=1}^{k} \bar{\lambda}_{i} \Phi\left(\bar{x}, \bar{y},\left(\xi_{i}, \rho_{i}\right)\right)<0
$$

holds for any $\xi_{i} \in \partial\left(f_{i}(\bar{y})-\bar{v}_{i} q_{i}(\bar{y})\right), i=1, \ldots, k$. Using $\bar{x} \in D,(\bar{y}, \bar{v}, \bar{\lambda}, \bar{\mu}, \bar{\omega}) \in \Gamma, b_{g_{j}}(\bar{x}, \bar{y})>0, i=1, \ldots, k$, together with hypothesis $b$ ), we get that the inequality

$$
\sum_{j \in J(\bar{y})} \bar{\mu}_{j} \Phi\left(\bar{x}, \bar{y},\left(\zeta_{j}, \rho_{g_{j}}\right)\right) \leqq 0
$$

holds for any $\zeta_{j} \in \partial g_{j}(\bar{y}), j \in J(\bar{y})$. Using hypotheses c) and d), by Definition 7 , the inequalities

$$
\begin{gathered}
b_{h_{s}}(\bar{x}, \bar{y}) \Psi_{h_{s}}\left(h_{s}(\bar{x})-h_{s}(\bar{y})\right) \geqq \Phi\left(\bar{x}, \bar{y},\left(\varsigma_{s}, \rho_{h_{s}}^{+}\right)\right), \quad s \in S^{+}(\bar{y}), \\
b_{h_{s}}(\bar{x}, \bar{y}) \Psi_{h_{s}}\left(-h_{s}(\bar{x})+h_{s}(\bar{y})\right) \geqq \Phi\left(\bar{x}, \bar{y},\left(-\varsigma_{s}, \rho_{h_{s}}^{-}\right)\right), \quad s \in S^{-}(\bar{y})
\end{gathered}
$$

hold for any $\varsigma_{s} \in \partial h_{s}(\bar{y}), s \in S^{+}(\bar{y})$ and $-\varsigma_{s} \in \partial\left(-h_{s}\right)(\bar{y}), s \in S^{-}(\bar{y})$, respectively. Multiplying (74) by $\bar{\omega}_{s}, s \in S^{+}(\bar{y}),(75)$ by $-\bar{\omega}_{s}, s \in S^{-}(\bar{y})$ and using the assumption that any $\Psi_{h_{s}}, s \in S^{+}(\bar{y}) \cup S^{-}(\bar{y})$ is a homogeneous function, we get

$$
\begin{gathered}
b_{h_{s}}(\bar{x}, \bar{y}) \Psi_{h_{s}}\left(\bar{\omega}_{s} h_{s}(\bar{x})-\bar{\omega}_{s} h_{s}(\bar{y})\right) \geqq \bar{\omega}_{s} \Phi\left(\bar{x}, \bar{y},\left(\varsigma_{s}, \rho_{h_{s}}^{+}\right)\right), \quad s \in S^{+}(\bar{y}), \\
b_{h_{s}}(\bar{x}, \bar{y}) \Psi_{h_{s}}\left(\bar{\omega}_{s} h_{s}(\bar{x})-\bar{\omega}_{s} h_{s}(\bar{y})\right) \geqq \bar{\omega}_{s} \Phi\left(\bar{x}, \bar{y},\left(-\varsigma_{s}, \rho_{h_{s}}^{-}\right)\right), \quad s \in S^{-}(\bar{y}) .
\end{gathered}
$$

By $\bar{x} \in D$ and $(\bar{y}, \bar{v}, \bar{\lambda}, \bar{\mu}, \bar{\omega}) \in \Gamma$, we have

$$
\bar{\omega}_{s} h_{s}(\bar{x})-\bar{\omega}_{s} h_{s}(\bar{y}) \leqq 0, \quad s \in S
$$

By hypotheses c) and d), (78) yields, respectively,

$$
b_{h_{s}}(\bar{x}, \bar{y}) \Psi_{h_{s}}\left(\bar{\omega}_{s} h_{s}(\bar{x})-\bar{\omega}_{s} h_{s}(\bar{y})\right) \leqq 0, \quad s \in S^{+}(\bar{y}) \cup S^{-}(\bar{y})
$$

Combining (76), (77), and (79) and then adding the resulting inequalities, we get that the inequalities

$$
\begin{gathered}
\sum_{s \in S^{+}(\bar{y})} \omega_{s} \Phi\left(x, \bar{y},\left(\varsigma_{s}, \rho_{h_{s}}^{+}\right)\right) \leqq 0 \\
\sum_{s \in S^{-}(\bar{y})}\left(-\omega_{s}\right) \Phi\left(x, \bar{y},\left(-\varsigma_{s}, \rho_{h_{s}}^{-}\right)\right) \leqq 0
\end{gathered}
$$

hold for any $\varsigma_{s} \in \partial h_{s}(\bar{y}), s \in S^{+}(\bar{y})$ and $-\varsigma_{s} \in \partial\left(-h_{s}\right)(\bar{y}), s \in S^{-}(\bar{y})$, respectively. By (72), (73), (80), and (81), we have that

$$
\sum_{i=1}^{k} \bar{\lambda}_{i} \Phi\left(\bar{x}, \bar{y},\left(\xi_{i}, \rho_{i}\right)\right)+\sum_{j \in J(\bar{y})} \bar{\mu}_{j} \Phi\left(\bar{x}, \bar{y},\left(\zeta_{j}, \rho_{g_{j}}\right)\right)+
$$




$$
\sum_{s \in S^{+}(\bar{y})} \omega_{s} \Phi\left(x, \bar{y},\left(\varsigma_{s}, \rho_{h_{s}}^{+}\right)\right)+\sum_{s \in S^{-}(\bar{y})}\left(-\omega_{s}\right) \Phi\left(x, \bar{y},\left(-\varsigma_{s}, \rho_{h_{s}}^{-}\right)\right)<0 .
$$

The rest of the proof of this theorem is similar to the proof of Theorem 16. The proof of this theorem under hypothesis B) is similar and, therefore, it has been omitted from the paper.

\section{Conclusion}

In this paper, the class of nonconvex nondifferentiable multiobjective fractional programming problems in which the involved functions are locally Lipschitz $(b, \Psi, \Phi, \rho)$-univex has been considered. For such nonsmooth vector optimization problems, the parametric optimality conditions and parametric duality results in the sense of Schaible have been established under the introduced notion of nondifferentiable $(b, \Psi, \Phi, \rho)$-univexity. Since the concept of nondifferentiable $(b, \Psi, \Phi, \rho)$-univexity contains several generalized convexities defined earlier in the literature (see Remark 8), the optimality and duality results established in the present paper generalize and extend similar results for multiobjective fractional programming problems presented in earlier works. It would be interesting to generalize the optimality conditions and duality results established in the present paper for

nonconvex multiobjective fractional programming problems to other classes of fractional programming problems. We shall investigate this question in subsequent papers.

\section{References}

[1] Ahmad I. Optimality conditions and duality in fractional minimax programming involving generalized $\rho$-invexity. Int J Man Sys 2003; 19: 165-180.

[2] Antczak T. Generalized fractional minimax programming with $B$ - $(p, r)$-invexity. Comput Math Appl 2008; 56: 1505-1525.

[3] Antczak T. On nonsmooth $(\Phi, \rho)$-invex multiobjective programming in finite-dimensional Euclidean spaces. J Adv Math Stud 2014; 7: 127-145.

[4] Antczak T, Stasiak A. $(\Phi, \rho)$-invexity in nonsmooth optimization. Numer Func Anal Opt 2011; 32 : 1-25.

[5] Bector CR, Chandra S, Bector MK. Generalized fractional programming duality: a parametric approach. J Optim Theory Appl 1989; 60: 243-260.

[6] Bector CR, Chandra S, Husain I. Optimality conditions and duality in subdifferentiable multiobjective fractional programming. J Optim Theory Appl 1993; 79: 105-125.

[7] Bector CR, Chandra S, Gupta S, Suneja SK. Univex sets, functions and univex nonlinear programming. In: Komolosi S, Rapcsák T, Schaible S, editors. Generalized convexity. Lecture Notes in Economics and Mathematical Systems Vol. 405. Berlin, Germany: Springer Verlag, 1994.

[8] Bhatia D, Garg PK. Duality for nonsmooth nonlinear fractional multiobjective programs via $(F, \rho)$-convexity. Optimization 1998; 43: 185-197.

[9] Chandra S, Craven BD, Mond B. Generalized fractional programming duality: a ratio game approach. J Aust Math Soc Ser B 1986; 28: 170-180.

[10] Chen X. Optimality and duality for the multiobjective fractional programming with the generalized $(F, \rho)$-convexity. J Math Anal Appl 2002; 273: 190-205.

[11] Crouzeix JP, Ferland JA, Schaible S. An algorithm for generalized fractional programs. J Optim Theory Appl 1985; 47: $35-49$,

[12] Chinchuluun A, Yuan D, Pardalos PM. Optimality conditions and duality for nondifferentiable multiobjective fractional programming with generalized convexity. Ann Oper Res 2007; 154: 133-147. 
ANTCZAK and VERMA/Turk J Math

[13] Clarke FH. Optimization and Nonsmooth Analysis. New York, NY, USA: John Wiley\&Sons, Inc., 1983.

[14] Craven BD. Kinds of vector invex. Taiwan J Math 2010; 14: 1925-1933.

[15] Hanson MA, Mond B. Further generalization of convexity in mathematical programming. J Inform Optimiz Sci 1982; 3: 25-32.

[16] Jayswal A, Kumar R, Kumar D. Minimax fractional programming problem involving nonsmooth generalized $\alpha$ univex functions. Int J Opt Control Theories Appl 2013; 3: 7-22.

[17] Jeyakumar V. Equivalence of saddle-points and optima, and duality for a class of nonsmooth non-convex problems. J Math Anal Appl 1988; 130: 334-343.

[18] Kim DS. Nonsmooth multiobjective fractional programming with generalized invexity. Taiwan J Math 2006; 10: 467-478.

[19] Kim DS, Schaible S. Optimality and duality for invex nonsmooth multiobjective programming problems. Optimization 2004; 53: 165-176.

[20] Kim GS, Kim MH, Lee GM. On optimality and duality for nonsmooth multiobjective fractional optimization problems. Nonlinear Anal 2005; 63: 1867-1876.

[21] Kuk H, Lee GL, Tanino T. Optimality and duality for nonsmooth multiobjective fractional programming with generalized invexity. J Math Anal Appl 2001; 262: 365-375.

[22] Lai HC, Ho SC. Optimality and duality for nonsmooth multiobjective fractional programming problems involving exponential $V$-r-invexity. Nonlinear Anal 2012; 75: 3157-3166.

[23] Lee G.M. Nonsmooth invexity in multiobjective programming. Journal of Information and Optimization Sciences 1994; 15: 127-136.

[24] Li XF, Dong JL, Liu QH. Lipschitz B-vex functions and nonsmooth programming. J Optim Theory Appl 1997; 93: 557-574.

[25] Liang ZA, Huang HX, Pardalos PM. Optimality conditions and duality for a class of nonlinear fractional programming problems. J Optim Theory Appl 2001; 110: 611-619.

[26] Liang ZA, Huang HX, Pardalos PM. Efficiency conditions and duality for a class of multiobjective fractional programming problems. J Global Optim 2003; 27: 447-471.

[27] Liu JC. Optimality and duality for multiobjectional fractional programming involving nonsmooth $(F, \rho)$-convex functions. Optimization 1996; 36: 333-346.

[28] Liu S. Feng E. Optimality conditions and duality for a class of nondifferentiable multi-objective fractional programming problems. J Global Optim 2007; 38: 653-666.

[29] Mishra SK. Generalized fractional programming problems containing locally subdifferentiable $\rho$-univex functions. Optimization 1997; 41: 135-158.

[30] Mishra SK, Upadhyay BB. Efficiency and duality in nonsmooth multiobjective fractional programming involving $\eta$-pseudolinear functions. Yugoslav J Oper Res 2012; 22: 3-18.

[31] Mishra SK, Wang SY, Lai KK. Multiple objective fractional programming involving semilocally type I -preinvex and related functions. J Math Anal Appl 2005; 310: 626-640.

[32] Mukherjee RN, Purnachandra Rao Ch. Generalized F-convexity and its classification. Indian J Pure Appl Math 1996; 27: 1175-1183.

[33] Nobakhtian S. Optimality and duality for nonsmooth multiobjective fractional programming with mixed constraints. J Global Optim 2008; 41: 103-115.

[34] Pandian P. On sufficiency and duality for $(b, F, \rho)$-convex multiobjective programs. Indian J Pure Appl Math 2002; 33: 463-473.

[35] Preda V. Optimality and duality in fractional multiple objective programming involving semilocally preinvex and related functions. J Math Anal Appl 2003; 288: 365-382. 
[36] Reiland TW. Nonsmooth invexity. Bull Aust Math Soc 1990; 42: 437-446.

[37] Soleimani-damaneh M. Optimality for nonsmooth fractional multiple objective programming. Nonlinear Anal 2008; 68: 2873-2878.

[38] Stancu-Minasian IM. A sixth bibliography of fractional programming. Optimization 2006; 55: $405-428$.

[39] Stancu-Minasian IM. Optimality and duality in fractional programming involving semilocally preinvex and related functions. J Inf Optim Sci 2002; 23: 185-201.

[40] Suneja SK, Lalitha CS. Multiobjective fractional programming involving $\rho$-invex and related functions. Opsearch 1993; 30: 1-14.

[41] Vial JP. Strong and weak convexity of sets and functions. Math Oper Res 1983; 8: 231-259.

[42] Weir T. A duality theorem for a multiobjective fractional optimization problem. B Aust Math Soc 1986; $34:$ 376-386.

[43] Zalmai GJ. Optimality conditions and duality models for generalized fractional programming problems containing locally subdifferentiable and $\rho$-convex functions. Optimization 1995; 32: 95-124.

[44] Zhang J, Mond B. Duality for a class of nondifferentiable fractional programming problems. Inter J Manag Syst 1998; 14: 71-88. 